\title{
Polycyclic aromatic hydrocarbon processing in a hot gas
}

\author{
E. R. Micelotta ${ }^{1,2}$, A. P. Jones ${ }^{2}$, and A. G. G. M. Tielens ${ }^{1,3}$ \\ 1 Sterrewacht Leiden, Leiden University, PO Box 9513, 2300 RA Leiden, The Netherlands \\ e-mail: micelot@strw.leidenuniv.nl \\ 2 Institut d'Astrophysique Spatiale, Université Paris Sud and CNRS (UMR 8617), 91405 Orsay, France \\ 3 NASA Ames Research Center, MS 245-3, Moffett Field, CA 94035, USA
}

Received 20 January 2009 / Accepted 16 October 2009

\section{ABSTRACT}

\begin{abstract}
Context. PAHs are thought to be a ubiquitous and important dust component of the interstellar medium. However, the effects of their immersion in a hot (post-shock) gas have never before been fully investigated.

Aims. We study the effects of energetic ion and electron collisions on PAHs in the hot post-shock gas behind interstellar shock waves. Methods. We calculate the ion-PAH and electron-PAH nuclear and electronic interactions, above the carbon atom loss threshold, in $\mathrm{H}$ II regions and in the hot post-shock gas for temperatures ranging from $10^{3}-10^{8} \mathrm{~K}$.

Results. PAH destruction is dominated by He collisions at low temperatures $\left(T<3 \times 10^{4} \mathrm{~K}\right)$, and by electron collisions at higher temperatures. Smaller PAHs are destroyed faster for $T<10^{6} \mathrm{~K}$, but the destruction rates are roughly the same for all PAHs at higher temperatures. The PAH lifetime in a tenuous hot gas $\left(n_{\mathrm{H}} \approx 0.01 \mathrm{~cm}^{-3}, T \approx 10^{7} \mathrm{~K}\right)$, typical of the coronal gas in galactic outflows, is found to be about thousand years, orders of magnitude shorter than the typical lifetime of such objects.

Conclusions. In a hot gas, PAHs are principally destroyed by electron collisions and not by the absorption of X-ray photons from the hot gas. The resulting erosion of PAHs occurs via $\mathrm{C}_{2}$ loss from the periphery of the molecule, thus preserving the aromatic structure. The observation of PAH emission from a million degree, or more, gas is only possible if the emitting PAHs are ablated from dense, entrained clumps that have not yet been exposed to the full effect of the hot gas.
\end{abstract}

Key words. shock waves - dust, extinction - ISM: jets and outflows

\section{Introduction}

The mid-infrared spectral energy distribution of the general interstellar medium of galaxies is dominated by strong and broad emission features at 3.3, 6.2, 7.7 and $11.3 \mu \mathrm{m}$. These features are now univocally attributed to vibrational fluorescence of UV pumped, large ( $\simeq 50 \mathrm{C}$-atoms) polycyclic aromatic hydrocarbon (PAHs) molecules. These large molecules are very abundant $\left(3 \times 10^{-7}\right.$ by number relative to $\mathrm{H}$-nuclei) and ubiquitous in the ISM (for a recent review see Tielens 2008). Besides large PAH molecules, the spectra also reveal evidence for clusters of PAHs - containing some hundreds of carbon atoms - and very small grains $(\simeq 30 \AA)$. Indeed, PAHs seem to represent the extension of the interstellar dust size distribution into the molecular domain (e.g. Désert et al. 1990; Draine \& Li 2001).

PAH molecules are an important component of the ISM, for example, dominating the photoelectric heating of neutral atomic gas and the ionization balance of molecular clouds. Small dust grains and PAHs can also be important agents in cooling a hot gas, at temperatures above $\sim 10^{6} \mathrm{~K}$ (e.g. Dwek 1987), through their interactions with thermal electrons and ions. The energy transferred in electron and ion collisions with the dust is radiated as infrared photons. The evolution of dust in such hot gas $(T \gtrsim$ $10^{6} \mathrm{~K}$ ), e.g., within supernova remnants and galactic outflows, is critical in determining the dust emission from these regions and therefore the cooling of the hot gas. The destruction of PAHs and small dust grains in a hot gas may also be an important process in the lifecycle of such species (Dwek et al. 1996; Jones et al. 1996).

Observationally, there is little direct evidence for PAH emission unequivocally connected to the hot gas in supernova remnants. Reach et al. (2006) have identified four supernova remnants with IR colors that may indicate PAH emission. Tappe et al. (2006) have detected spectral structure in the emission characteristics of the supernova remnant N132D in the Large Magellanic Cloud that they attribute to spectral features of PAHs with sizes of $\simeq 4000 \mathrm{C}$-atoms. Bright $8 \mu \mathrm{m}$ emission has been observed by IRAC/Spitzer associated with the X-ray emission from the stellar winds of the ionizing stars in the $\mathrm{M} 17 \mathrm{H}$ II region (Povich et al. 2007). Likely, this emission is due to PAHs - probably, in entrained gas ablated from the molecular clouds to the north and west of the stellar cluster. Finally, bright PAH emission has been detected associated with the hot gas of the galactic wind driven by the starburst in the nucleus of the nearby irregular galaxy, M 82 (Engelbracht et al. 2006; Beirão et al. 2008; Galliano et al. 2008).

Electron and ion interactions with dust and the implications of those interactions for the dust evolution and emission have already been discussed in the literature (e.g. Draine \& Salpeter 1979; Dwek 1987; Jones et al. 1994; Dwek et al. 1996; Jones et al. 1996). In this work we extend this earlier work to the case for PAHs, using our study of PAH evolution due to ion and electron interactions in shock waves in the ISM (Micelotta et al. 2010, hereafter MJT). Here we consider the fate of PAHs in the hot gas behind fast non-radiative shocks and in a hot gas in general.

The aim of this paper is to study the PAH stability against electron and ion collisions $(\mathrm{H}, \mathrm{He}$ and $\mathrm{C})$ in a thermal gas with temperature $T$ in the range $10^{3}-10^{8} \mathrm{~K}$.

The paper is organized as follows: Sects. 2 and 3 describe the treatment of ion and electron interactions with PAHs, Sect. 4 illustrates the application to PAH processing in a hot gas and 
Sect. 5 presents our results on PAH destruction and lifetime. The astrophysical implications are discussed in Sect. 6 and our conclusions summarized in Sect. 7.

\section{Ion interaction with PAHs}

\subsection{Electronic interaction}

The ion - PAH collision can be described in terms of two simultaneous processes which can be treated separately (Lindhard et al. 1963): nuclear stopping or elastic energy loss and electronic stopping or inelastic energy loss.

The nuclear stopping consists of a binary collision between the incoming ion (projectile) and a single atom in the target material. A certain amount of energy will be transferred directly to the target atom, which will be ejected if the energy transferred is sufficient to overcome the threshold for atom removal. The physics of the nuclear interaction for a PAH target was presented in the companion paper (MJT). A summary of the theory is provided in Sect. 2.2 and we here present the results of our calculation.

In this paper we focuse on the electronic stopping, which consists of the interaction between the projectile and the electrons of the target $\mathrm{PAH}$, with a subsequent energy transfer to the whole molecule. The resulting electronic excitation energy will be transferred to the molecular vibrations of the PAH through radiationless processes (e.g., interconversion \& intramolecular vibrational redistribution). The vibrationally excited molecule will decay through either (IR) photon emission or through fragmentation (i.e., $\mathrm{H}$-atom or $\mathrm{C}_{2} \mathrm{H}_{n}$ loss, where $n=0,1,2$ ).

No specific theory describes the energy transfer to a $\mathrm{PAH}$ via electronic excitation, so we adopt the same approach developed by e.g. Schlathölter et al. (1999) and Hadjar et al. (2001) who modelled electronic interactions in fullerene, $\mathrm{C}_{60}$. To calculate the energy transferred to a PAH, we treat the large number of delocalized valence electrons in the molecule as an electron gas, where the inelastic energy loss of traveling ions is due to long range coupling to electron-hole pairs (Ferrell 1979).

In the energy range we consider for this study, the energy transferred scales linearly with the velocity $v$ of the incident ion and can be described in term of the stopping power $S$, which is widely used in the treatment of ion-solid collisions. The stopping power represents the energy loss per unit length and is defined as

$S=\frac{\mathrm{d} T}{\mathrm{~d} s}=-\gamma\left(r_{s}\right) v$

where $\mathrm{d} T$ is the energy loss over the pathlength $\mathrm{d} s$ (Sigmund 1981). The total energy loss can then be obtained integrating Eq. (1)

$T_{\mathrm{e}}=\int \gamma\left(r_{s}\right) v \mathrm{~d} s$

The friction coefficient $\gamma$ (Puska \& Nieminen 1983) depends on the density parameter $r_{s}=\left(\frac{4}{3} \pi n_{0}\right)^{-1 / 3}$ which is a function of the valence electron density $n_{0}$. The choice of the valence electron density is a delicate issue for PAHs. In the case of fullerene, this is assumed to be the spherically symmetric jellium shell calculated by Puska \& Nieminen (1993), which can be approximated by the following expression (Hadjar et al. 2001):

$n_{0}=0.15 \exp \left[-(r-6.6)^{2} / 2.7\right]$

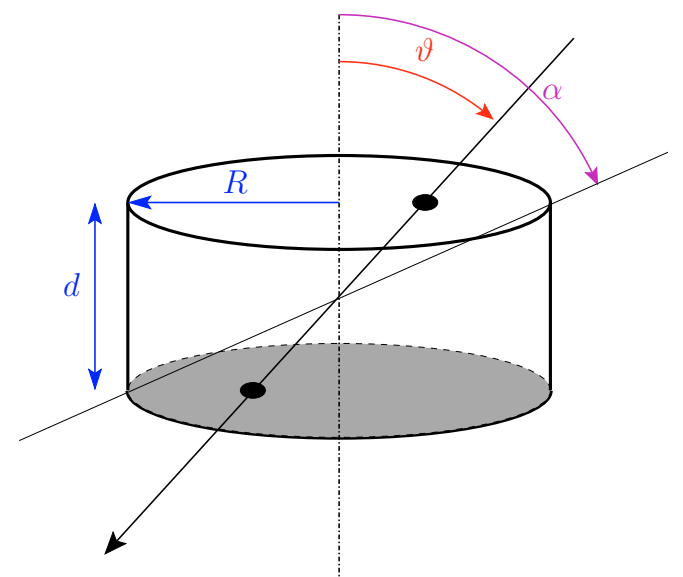

Fig. 1. The coordinate system adopted to calculate the energy transferred to a PAH via electronic excitation by ion collisions and by impacting electrons. The molecule is modeled as a disk with radius $R$ and thickness $d$. The trajectory of the incoming particle is identified by the angle $\vartheta$, while the angle $\alpha$ corresponds to the diagonal of the disk.

where $r$ (in atomic units, a.u. ${ }^{1}$ ) is the distance from the fullerene center. The electron density decays outside the shell and toward the center in such a way that the major contribution comes from the region with $4<r<9$.

The similarity in $\pi$ electronic structure and bonding allows us to apply this jellium model also to PAHs. However, the spherical geometry is clearly not appropriate for PAHs, which we model instead as a thick disk analogously to the distribution from Eq. (3)

$n_{0}=0.15 \exp \left[-(x)^{2} / 2.7\right]$

where $x$ is the coordinate along the thickness of the disk. This electron density peaks at the center of the molecule and vanishes outside, leading to a thickness $d \sim 4.31 \AA$. The radius $R$ of the disk is given by the usual expression for the radius of a PAH: $\left.a_{\mathrm{PAH}}=R=0.9 \sqrt{(} N_{\mathrm{C}}\right) \AA$, where $N_{\mathrm{C}}$ is the number of carbon atoms in the molecule (Omont 1986). For a 50 C-atoms PAH, $R=6.36 \AA$

To calculate the energy transferred from Eq. (2), we adopt the coordinate system shown in Fig. 1, where the pathlength through the PAH $s$ is expressed as a function of the coordinate $x$ and of the angle $\vartheta$ between the axes of the molecule and the direction of the incoming ion. In this way for each trajectory given by $\vartheta$ the corresponding energy transferred can be computed. We have $\mathrm{d} x=\mathrm{d} s \cos \vartheta$ and the electron density is then given by

$n_{0}(s, \vartheta)=0.15 \exp \left[-(s \cos \vartheta)^{2} / 2.7\right]$.

The density parameter can be rewritten as $r_{s}(s, \vartheta)=$ $\left(\frac{4}{3} \pi n_{0}(s, \vartheta)\right)^{-1 / 3}$ The friction coefficient $\gamma$ has been calculated by Puska \& Nieminen (1983) for various projectile ions and $r_{s}$ values. It can be interpolated by the exponential function

$\gamma\left(r_{s}\right)=\Gamma_{0} \exp \left[\frac{-\left(r_{s}(s, \vartheta)-1.5\right)}{R_{2}}\right]$

where $\Gamma_{0}$ is the value of $\gamma$ when $r_{s}=1.5$. For hydrogen, helium and carbon the fit parameter $R_{2}$ equals $2.28,0.88$ and 0.90 , respectively.

\footnotetext{
${ }^{1}$ Length (a.u.) $=a_{0}=0.529 \AA$, Velocity (a.u.) $=0.2 \sqrt{E(\mathrm{keV} / \mathrm{amu})}$, Energy (a.u.) $=27.2116 \mathrm{eV}$.
} 
The energy transferred is then given by the following equation

$T_{\mathrm{e}}(\vartheta)=27.2116 \int_{-R / \sin \vartheta}^{R / \sin \vartheta} v \gamma\left(r_{s}\right) \mathrm{d} s$

with $\gamma\left(r_{s}\right)$ from Eq. (6). The constant 27.2116 converts the dimensions of $T_{\mathrm{e}}$ from atomic units to $\mathrm{eV}$. For a given velocity of the incident particle, the value of $T_{\mathrm{e}}$ will be maximum for $\vartheta=\pi / 2(s=2 R$, the longest pathlength) and minimum for $\vartheta=0, \pi(s=d$, the shortest pathlength). The deposited energy also increases with the pathlength across the molecule, and then will be higher for larger PAHs impacted under a glancing collision.

The energy transferred determines the PAH dissociation probability upon electronic excitation, which is required to quantify the destruction induced by inelastic energy loss in the hot gas (see Sect. 4).

\subsection{Nuclear interaction above threshold}

In the present study we have to consider not only the electronic interaction described above, but the nuclear part of the ionic collision as well. The theory of nuclear interaction above threshold has been described in detail in MJT. We summarize here for clarity the essential concepts and the equations which will be used in the following.

For the nuclear interaction we consider only collisions above threshold, i.e. collisions able to transfer more than the minimum energy $T_{0}$ required to remove a C-atom from the PAH. To calculate the PAH destruction due to nuclear interaction, we use the rate of collisions above threshold between PAHs and ions in a thermal gas, as given by Eq. (30) in MJT:

$R_{\mathrm{n}, \mathrm{T}}=N_{\mathrm{C}} 0.5 \chi_{i} n_{\mathrm{H}} \int_{v_{0}}^{\infty} F_{\mathrm{C}} v \sigma(v) f(v, T) \mathrm{d} v$

where $f(v, T)$ is the Maxwellian velocity distribution function for ion, $i$, in the gas, $F_{\mathrm{C}}$ is the Coulombian correction factor, $\sigma(v)$ is the nuclear interaction cross section per atom above threshold (Eq. (22) in MJT where $E$ is the kinetic energy corresponding to $v$ ), and the factor $1 / 2$ takes the averaging over the orientation of the disk into account.

For $T_{0}$ we adopt a value of $7.5 \mathrm{eV}$ (see discussion in MJT, Sect. 2.2.1). The kinetic energy required for the incoming ion to transfer $T_{0}$ is the critical energy $E_{0}$. The lower integration limit $v_{0}$ in Eq. (8) is the critical velocity corresponding to $E_{0 \mathrm{n}}$, which is the minimum kinetic energy for the projectile to have the nuclear interaction cross section different from zero (cf. Sect. 2.2 in MJT).

\section{Electron collisions with PAHs}

Fast electrons are abundant in a hot gas. Because of their low mass, they can reach very high velocities with respect to the ions, and hence high rates of potentially destructive collisions. We consider gas temperatures up to $10^{8} \mathrm{~K}$, corresponding to a thermal electron energy of $\sim 10 \mathrm{keV}$, well below the relativistic limit of $\sim 500 \mathrm{keV}$. Under these low-energy conditions, with respect to the relativistic regime, elastic collisions between electrons and target nuclei are not effective. The energy transfer occurs through inelastic interactions with target electrons (as for electronic excitation by impacting ions), which lead to a collective excitation of the molecule, followed eventually by dissociation or relaxation through IR emission.
Table 1. Analytical fit to the electron stopping power in solid carbon.

\begin{tabular}{cccc}
\hline \hline \multicolumn{4}{c}{$S(E)=h \log (1+a E) / f E^{g}+b E^{d}+c E^{e}$} \\
\hline$a$ & $b$ & $c$ & $d$ \\
-0.000423375 & $-3.57429 \times 10^{-11}$ & $-3.37861 \times 10^{-7}$ & -3.18688 \\
\hline$e$ & $f$ & $g$ & $h$ \\
-0.587928 & -0.000232675 & 1.53851 & 1.41476 \\
\hline
\end{tabular}

The calculation of the energy transferred by such "slow" electrons is in fact a delicate matter. The theory developed under the first Born approximation (Bethe 1930) can be applied only to the most energetic electrons (around few $\mathrm{keV}$ ) but is unsuitable for the rest of our range. At low energies $(<10 \mathrm{keV})$, where the first Born approximation is no longer valid, the Mott elastic cross section must be used instead of the conventional Rutherford cross section (Mott \& Massey 1949; Czyzewski et al. 1990). The Monte Carlo program CASINO (Hovington et al. 1997) computes the Mott cross sections in the simulation of electron interactions with various materials. Unfortunately the stopping power $\mathrm{d} E / \mathrm{d} x$ is not included in the program output. An empirical expression for $\mathrm{d} E / \mathrm{d} x$ has been proposed by Joy \& Luo (1989), which nevertheless is reliable only down to $50 \mathrm{eV}$, while we are interested in the region between 10 and $50 \mathrm{eV}$ as well.

We decided thus to derive the electron stopping power from experimental results. Measurements of the electron energy loss in PAHs are not available in our energy range of interest, so we use the measurement of $\mathrm{d} E / \mathrm{d} x$ in solid carbon for electrons with energy between $10 \mathrm{eV}$ and $2 \mathrm{keV}$ (Joy 1995). The data points are well fitted (to within few \%) by the following function:

$S(E)=\frac{h \log (1+a E)}{f E^{g}+b E^{d}+c E^{e}}$

where $E$ is the electron energy (in $\mathrm{keV}$ ). The values of the fitting parameters are reported in Table 1. $S(E)$ has the same functional form as the ZBL reduced stopping cross section for nuclear interaction (cf. Sect. 2.1 in MJT). The datapoints and the fitting function are shown in the top panel of Fig. 2.

The stopping power increases sharply at low electron energies, reaches its maximum at $\sim 0.1 \mathrm{keV}$, and decreases smoothly afterwards. Between 0.01 and $0.1 \mathrm{keV}$ a small variation of the energy of the incident electron will translate into a large change in the transferred energy per unit length. The shape of $S(E)$ implies that only those electrons with energies that fall in a well defined window will efficiently transfer energy, while electrons below $\sim 0.02 \mathrm{keV}$ and above $\sim 2 \mathrm{keV}$ are not expected to contribute significantly to PAH excitation.

Once the stopping power $\mathrm{d} E / \mathrm{d} x$ is known, we can calculate the energy transferred by an electron of given energy when travelling through the PAH. We adopt the same configuration used for electronic interaction, shown in Fig. 1. The trajectory of the incoming electron is defined by its impact angle $\vartheta$ and by the geometry of the molecule.

Because the thickness of the PAH is non-negligible with respect to its radius, the stopping power is not constant along the electron path. To calculate the energy loss we thus follow the procedure described below. We have $\mathrm{d} E / \mathrm{d} x=-S(E)$, then

$\int \mathrm{d} x=-\int \frac{\mathrm{d} E}{S(E)}=F(E)$.

Thus, $x_{1}-x_{0}=-\left[F\left(E_{1}\right)-F\left(E_{0}\right)\right]$ where $x_{1}-x_{o}=l(\vartheta)$ is the maximum pathlength through the PAH. From inspection of 

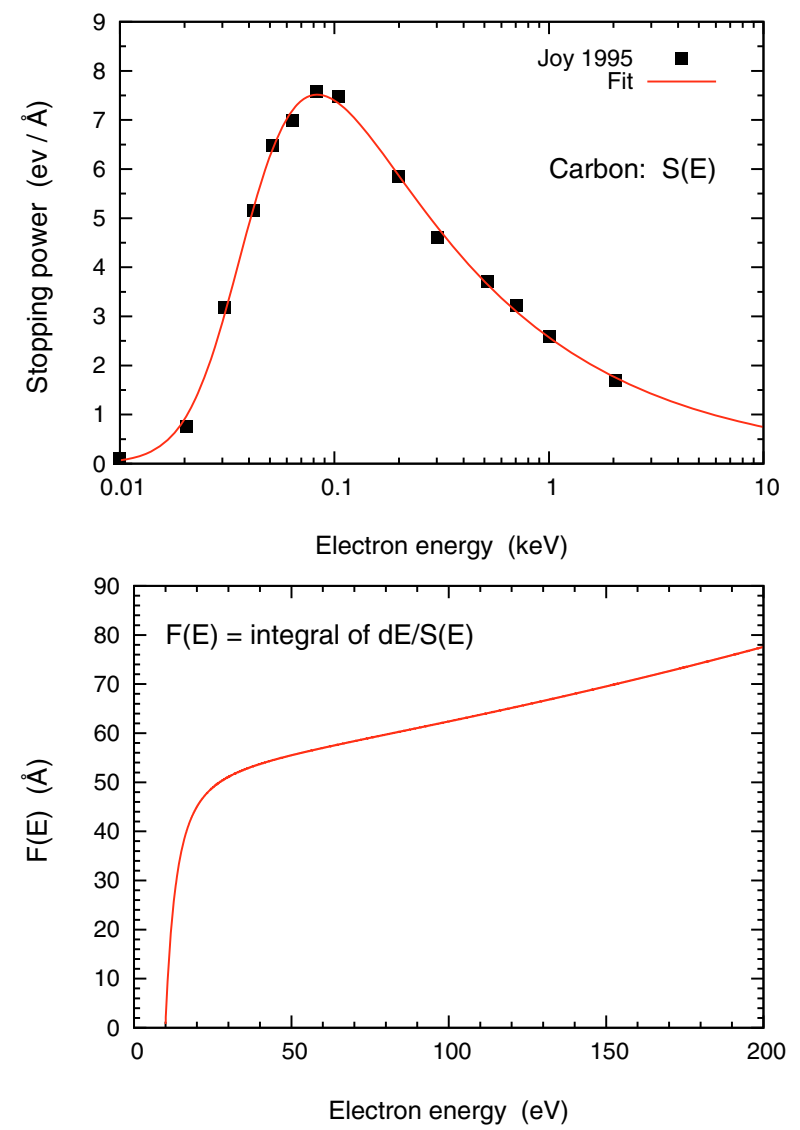

Fig. 2. Top panel - Experimental measurement of $\mathrm{d} E / \mathrm{d} x$ in solid carbon for electrons with energy between $10 \mathrm{eV}$ and $2 \mathrm{keV}$, from Joy (1995), overlaid with the fitting function $S(E)$ (solid line). Bottom panel Integral $F$ calculated numerically as a function of the energy of the incident electron, $E$.

Fig. 1, one can see that, if $|\tan (\vartheta)|<\tan (\alpha), l(\vartheta)=d /|\cos \vartheta|$, otherwise $l(\vartheta)=2 R /|\sin \vartheta| . E_{0}$ is the initial energy of the impacting electron, $E_{1}$ is the electron energy after having travelled the distance $l(\vartheta)$, which needs to be calculated so we can then determine the energy transferred to the PAH

$T_{\text {elec }}(\vartheta)=E_{0}-E_{1}$

The integral $F$, calculated numerically as a function of $E$, is shown in the bottom panel of Fig. 2. We recognize that for low energies, $F$ rises sharply, reflecting the small energy stopping power in this energy range (cf., top panel in Fig. 2). For higher energies, $F(E)$ rises slowly (and linearly) with increasing energy over the relevant energy range. We note that the initial rise depends strongly on the (uncertain) details of the stopping power at low energies. However, it is of no consequence in our determination of the amount of energy deposited since we are only concerned with those collisions for which the energy deposition is in excess of the threshold energy ( $\gtrsim 10 \mathrm{eV})$ over a pathlength of $\simeq 10-20 \AA$. Or to phrase it differently, at low energies a much larger pathlength has to be traversed (than is relevant for PAHs) in order to transfer sufficient energy to cause fragmentation. Then, since we know $E_{0}$, we can calculate $F\left(E_{0}\right)$, and $F\left(E_{1}\right)$ is then given by $F\left(E_{0}\right)-l(\vartheta)$. We also calculated $E$ as a function of $F(E)$. We can then determine $E_{1}$ from $F\left(E_{1}\right)$ and, finally, $T_{\text {elec }}$ from Eq. (11).

\section{PAH destruction}

\subsection{Dissociation probability}

Ion or electronic collisions (or UV photon absorption) can leave the molecule internally (electronically) excited with an energy $T_{\mathrm{E}}$. Internal conversion transfers this energy to vibrational modes, and the molecule can then relax through dissociation or IR emission. These two processes are in competition with each other. To quantify the PAH destruction due to ion and electron collisions we need to determine the probability of dissociation rather than IR emission.

In the microcanonical description of a PAH, the internal energy, $T_{\mathrm{E}}$, is (approximately) related to the effective temperature of the system, $T_{\text {eff }}$, by the following equation

$T_{\text {eff }} \simeq 2000\left(\frac{T_{\mathrm{e}}(\mathrm{eV})}{N_{\mathrm{C}}}\right)^{0.4}\left(1-0.2 \frac{E_{0}(\mathrm{eV})}{T_{\mathrm{e}}(\mathrm{eV})}\right)$

where $E_{0}$ is the binding energy of the fragment (Tielens 2005). This effective temperature includes a correction for the finite size of the heat bath in the PAH. The temperature $T_{\text {eff }}$ is defined through the unimolecular dissociation rate, $k_{\text {diss }}$, written in Arrhenius form

$k_{\text {diss }}=k_{0}\left(T_{\text {eff }}\right) \exp \left[-E_{0} / k T_{\text {eff }}\right]$

where $k=8.617 \times 10^{-5} \mathrm{eV} / \mathrm{K}$ is the Boltzmann's constant (cf. Tielens 2005).

Consider the competition between photon emission at a rate $k_{\mathrm{IR}}$ (photons $\mathrm{s}^{-1}$ ) and dissociation at a rate of $k_{\text {diss }}$ (fragments $\mathrm{s}^{-1}$ ). For simplicity, we will assume that all photons have the same energy, $\Delta \varepsilon$. The probability that the PAH will fragment between the $n$th and $(n+1)$ th photon emission is given by

$\varphi_{n}=p_{n+1} \prod_{i=0}^{n}\left(1-p_{i}\right)$.

The (un-normalized) probability per step $p_{i}$ is given by $p_{i}=$ $k_{\text {diss }}\left(E_{i}\right) / k_{\mathrm{IR}}\left(E_{i}\right)$ and $E_{i}=\left(T_{\mathrm{E}}-i \times \Delta \varepsilon\right)$, with $T_{\mathrm{E}}$ the initial internal energy, coincident with the energy transferred into the PAH. The total dissociation probability is then given by

$P\left(n_{\max }\right)=\sum_{n=0}^{n_{\max }} \varphi_{n}$.

If we ignore the dependence of $k_{\mathrm{IR}}$ on $E_{i}$, the temperatures (Eq. (12)) drop approximately by $T_{i} / T_{i-1}=\left(1-0.4 \Delta \varepsilon / T_{\mathrm{E}}\right)$. The probability ratio is approximately:

$\frac{p_{i}}{p_{i-1}}=\exp \left[\left(\frac{E_{0}}{k T_{i-1}}\right) 0.4 \frac{\Delta \varepsilon}{\varepsilon}\right]$.

These equations become very difficult to solve in closed form. However, let us just assume that $p_{i}$ does not vary and is given by $p_{\mathrm{av}}$. Then we have the total un-normalized dissociation probability

$P\left(n_{\max }\right)=\left(n_{\max }+1\right) p_{\mathrm{av}}=\frac{k_{0} \exp \left[-E_{0} / k T_{\mathrm{av}}\right]}{k_{\mathrm{IR}} /\left(n_{\max }+1\right)}$

where we adopt $k_{\mathrm{IR}}=100$ photons $\mathrm{s}^{-1}$ (Jochims et al. 1994), and the average temperature is chosen as the geometric mean

$T_{\mathrm{av}}=\sqrt{T_{0} \times T_{n_{\max }}}$

where $T_{0}$ and $T_{n_{\max }}$ are the effective temperatures corresponding to $T_{\mathrm{E}}$ (initial internal energy equal to the energy transferred) and 
$\left(T_{\mathrm{E}}-n_{\max } \times \Delta \varepsilon\right.$ ) (internal energy after the emission of $n_{\max }$ photons). For the energy of the emitted IR photon we adopt the value $\Delta \varepsilon=0.16 \mathrm{eV}$, corresponding to a typical CC mode.

If the $p_{i}$ 's were truly constant, then $n_{\max }$ would be $n_{\max }=$ $\left(T_{\mathrm{E}}-E_{0}\right) / \Delta \varepsilon$. However, they do decrease. So, rather we take it to be when the probability per step has dropped by a factor 10 . A direct comparison between the full evaluation and this simple approximation yields $n_{\max }=10,20$ and 40 for $N_{\mathrm{C}}=50,100$ and 200 respectively. The quantity $n_{\max }$ scales with $N_{C}$ because for a constant temperature (e.g., required to get the dissociation to occur), the internal energy has to scale with $N_{\mathrm{C}}$. As a result, the number of photons to be emitted also has to scale with $N_{\mathrm{C}}$.

The choice of the values to adopt for $k_{0}$ and $E_{0}$ is a delicate matter. In the laboratory the dissociation of highly vibrationally excited PAHs is typically measured on timescales of $1-100 \mu \mathrm{s}$ because either the molecules are collisionally de-excited by ambient gas or the molecules have left the measurement zone of the apparatus. In contrast, in the ISM, the competing relaxation channel is through IR emission and occurs typically on a timescale of $1 \mathrm{~s}$. As is always the case for reactions characterized by an Arrhenius law, a longer timescale implies that the internal excitation energy can be lower. This kinetic shift is well established experimentally and can amount to many eV. Moreover, only small PAHs (up to $24 \mathrm{C}$-atoms) have been measured in the laboratory and the derived rates have to be extrapolated to much larger ( $\sim 50 \mathrm{C}$-atoms) PAHs that are astrophysically relevant. In an astrophysical context, the unimolecular dissociation of highly vibrationally excited PAHs - pumped by FUV photons - has been studied experimentally by Jochims et al. (1994) and further analyzed by Le Page et al. (2001). Here, we will modify the analysis of Tielens (2005) for H-loss by UV pumped PAHs to determine the parameters for carbon loss. The dissociation rate - given by Eq. (13) - is governed by two factors, the pre-exponential factor $k_{0}$ and the energy $E_{0}$. The pre-exponential factor is given by

$k_{0}=\frac{k T_{\text {eff }}}{h} \exp \left[1+\frac{\Delta S}{R}\right]$

where $\Delta S$ is the entropy change which we set equal to 10 cal mole ${ }^{-1}$ (Ling \& Lifshitz 1998). We will ignore the weak temperature dependence of $k_{0}$ in the following and adopt $1.4 \times 10^{16} \mathrm{~s}^{-1}$. The parameter $E_{0}$ can now be determined from a fit to the experiments by Jochims et al. (1994) on small PAHs. These experiments measured the appearance energy $E_{\text {ap }}-$ the internal energy at which noticable dissociation of the PAH first occurred. The rate at which this happened was assumed to be $10^{4} \mathrm{~s}^{-1}$. Adopting this value, the appearance energy can be estimated from Eq. (13). The results of our analysis are shown in Fig. 3. The internal energy required to dissociate a PAH depends strongly on the PAH size. Likewise the kinetic shift associated with the relevant timescale at which the experiment was performed is quite apparent. Indeed, this kinetic shift can amount to tens of eV for relevant PAHs.

The derived Arrhenius energy of $3.65 \mathrm{eV}$ is small compared to the binding energy of a $\mathrm{C}_{2} \mathrm{H}_{2}$ group in a PAH (4.2 eV, Ling \& Lifshitz 1998). This is a well known problem in statistical unimolecular dissociation theories (cf. Tielens 2008). We emphasize that these results show that a typical interstellar PAH with a size of $50 \mathrm{C}$-atoms would have a dissociation probability of $\sim 1 / 2$ after absorption of an FUV photon of $\sim 12 \mathrm{eV}$ (cf. Fig. 3). Hence, PAHs would be rapidly lost in the ISM through photolysis. It seems that the experiments on small PAHs cannot be readily extrapolated to larger, astrophysically relevant PAHs. Possibly, this

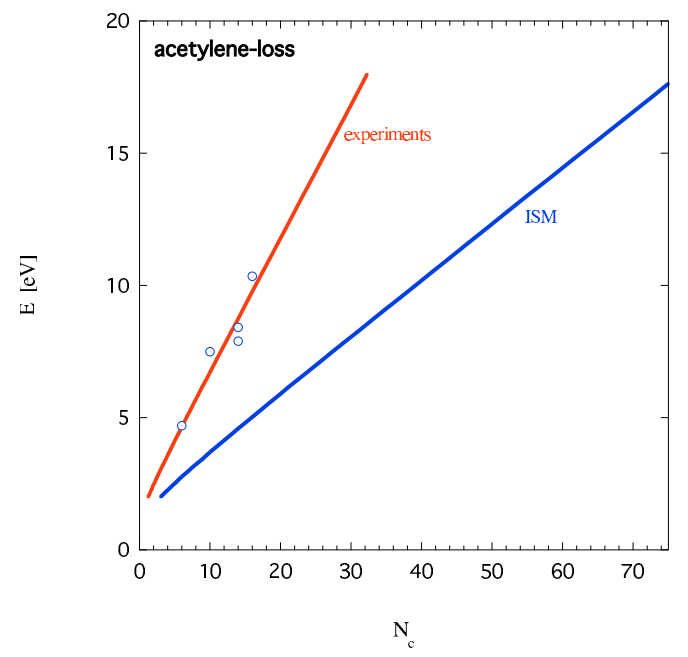

Fig. 3. The appearance energy as a function of the number of $\mathrm{C}$-atoms in the PAH. The red line provides a fit to the experimental data using Eq. (13) for an assumed pre-exponential factor $k_{0}=1.4 \times 10^{16}$ and a (fitted) Arrhenius energy, $E_{0}=3.65 \mathrm{eV}$. The data points are the experimental results of Jochims et al. (1994). The blue line is the appearance energy for ISM conditions (e.g., at a rate of $1 \mathrm{~s}^{-1}$ ).

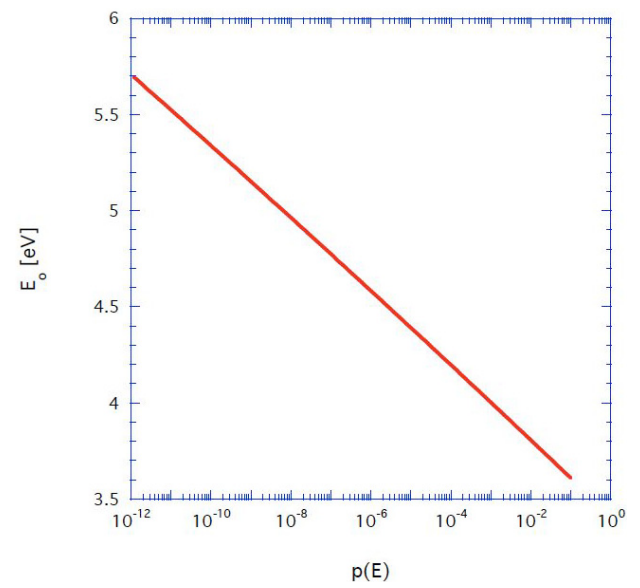

Fig. 4. The probability for dissociation of a $50 \mathrm{C}$-atom $\mathrm{PAH}$ excited by $10 \mathrm{eV}$ as a function of the Arrhenius energy, $E_{0}$.

is because experimentally $\mathrm{C}_{2} \mathrm{H}_{2}$ loss has only been observed for very small catacondensed PAHs with a very open carbon skeleton (e.g., naphthalene, anthracene, and phenanthrene) which are likely much more prone to dissociation than the astrophysically more relevant pericondensed PAHs. Indeed, the small pericondensed PAHs, pyrene and coronene did not show any dissociation on the experimental timescales (Jochims et al. 1994; Ling \& Lifshitz 1998).

Turning the problem around, we can determine the Arrhenius energy, $E_{0}$, as a function of the dissociation probability by adopting an IR relaxation rate of $1 \mathrm{~s}^{-1}$ and an internal excitation energy equal to a typical FUV photon energy $(12 \mathrm{eV})$. The results for a $50 \mathrm{C}$-atom PAH are shown in Fig 4. If we adopt a lifetime, $\tau_{\mathrm{PAH}}$, of 100 million years, a PAH in the diffuse ISM will have typically survived some $\sigma_{\mathrm{uv}} N_{\mathrm{uv}} \tau_{\mathrm{PAH}}=2 \times 10^{6} N_{\mathrm{C}} \mathrm{UV}$ photon absorptions (with $\sigma_{\mathrm{uv}}=7 \times 10^{-18} \mathrm{~cm}^{2}, N_{\mathrm{uv}}=10^{8}$ photons $\mathrm{cm}^{-2} \mathrm{~s}^{-1}$ in a Habing field). Hence, if the lifetime of the smallest PAHs in the ISM (e.g., with $N_{\mathrm{C}} \simeq 50 \mathrm{C}$-atom) is set by photodissociation of the $\mathrm{C}$-skeleton, the probability for dissociation has to be $5 \times 10^{-7}$ corresponding to an Arrhenius energy of $4.6 \mathrm{eV}$ (Fig. 4). We note that in a PDR the photon flux is 
higher $\left(G_{0} \sim 10^{5}\right)$ while the lifetime (of the PDR) is smaller $\left(\tau_{\mathrm{PDR}} \simeq 3 \times 10^{4} \mathrm{yr}\right)$, resulting in $6 \times 10^{7} \mathrm{UV}$ photons absorbed over the PDR lifetime. Survival of PAHs in a PDR environment would therefore require a somewhat larger $E_{0}$ (or alternatively, only slightly larger PAHs could survive in such an environment).

We note that the binding energy of a $\mathrm{C}_{2} \mathrm{H}_{2}$ group to small PAHs is estimated to be $4.2 \mathrm{eV}$ and is probably somewhat larger for a $50 \mathrm{C}$-atom condensed PAH. Loss of pure carbon, on the other hand, requires an energy of $7.5 \mathrm{eV}$, close to the binding energy of $\mathrm{C}$ to graphite. Loss of $\mathrm{C}_{2}$ from fullerenes has a measured $E_{0}$ of $9.5 \pm 0.1 \mathrm{eV}$ (Tomita et al. 2001). These latter two unimolecular dissociation channels are for all practical purposes closed under interstellar conditions. Finally, likely, $\mathrm{H}$-loss will be the dominant destruction loss channel for large PAHs $\left(E_{0}=3.3 \mathrm{eV}\right)$, leading to rapid loss of all H's (Le Page et al. 2001; Tielens 2005). The resulting pure C-skeleton may then isomerize to much more stable carbon clusters, in particular fullerenes, and this may be the dominant "loss" channel for interstellar PAHs (cf. Tielens 2008, and references therein). It is clear that there are many uncertainties in the chemical destruction routes of interstellar PAHs and that these can only be addressed by dedicated experimental studies. For now, in our analysis of the unimolecular dissociation of PAHs - excited by electron or ion collisions - we will adopt $E_{0}=4.6 \mathrm{eV}$ as a standard value. We will however also examine the effects of adopting $E_{0}=3.65 \mathrm{eV}$ (e.g., $p=1 / 2$ ) and $E_{0}=5.6 \mathrm{eV}$ (e.g., $\left.p=3 \times 10^{-12}\right)$.

\subsection{Collision rate}

Once the dissociation probability is determined, we can calculate the destruction rate through electronic excitation following electron or ion collision. Adopting the configuration shown in Fig. 1, the destruction rate is given by

$R_{\mathrm{e}}=v \chi n_{\mathrm{H}} F_{\mathrm{C}} \frac{1}{2 \pi} \int \mathrm{d} \Omega \sigma_{\mathrm{g}}(\vartheta) P(v, \vartheta)$

with $\Omega=\sin \vartheta \mathrm{d} \vartheta \mathrm{d} \varphi, \varphi$ running from 0 to $2 \pi$ and $\vartheta$ from 0 to $\pi / 2$. Equation (20) can be re-written as

$R_{\mathrm{e}}=v \chi n_{\mathrm{H}} F_{\mathrm{C}} \int_{\vartheta=0}^{\pi / 2} \sigma_{\mathrm{g}}(\vartheta) P(v, \vartheta) \sin \vartheta \mathrm{d} \vartheta$

The term $v$ is the velocity of the incident particle, $\chi$ is the ion/electron relative abundance in the gas, and $F_{\mathrm{C}}$ is the Coulombian correction factor, taking into account the fact that both target and projectiles are charged, and thus the collision cross section could be enhanced or diminished depending on the charge sign. The electron coulombian factor is always equal to 1 (to within 1\%) because electrons have low mass. For a fixed temperature they reach higher velocities, with respect to the ions, and are thus less sensitive to the effects of the Coulombian field. The quantity $\vartheta$ is the angle between the vertical axes of the PAH and the direction of the projectile. The term $\sigma_{\mathrm{g}}$ is the geometrical cross section seen by an incident particle with direction defined by $\vartheta$. The PAH is modelled as a thick disk with radius $R$ and thickness $d$. The cross section is then given by

$\sigma_{g}=\pi R^{2} \cos \vartheta+2 R d \sin \vartheta$

which reduces to $\sigma_{g}=\pi R^{2}$ for $\vartheta=0$ (face-on impact) and to $\sigma_{g}=2 R d$ for $\vartheta=\pi / 2$ (edge-on impact). The term $P(v, \vartheta)$ represents the total probability for dissociation upon electron collisions and electronic excitation, for a particle with velocity $v_{\mathrm{PAH}}$ and incoming direction $\vartheta$. It was calculated from Eq. (17) using the appropriated value of the energy transferred: $T_{\text {elec }}$ for electrons (Eq. (11)) and $T_{\mathrm{e}}$ for electronic excitation (Eq. (7)).

We are considering a hot gas and therefore we are interested in the thermal collision rate, given by

$R_{\mathrm{e}, \mathrm{T}}=\int_{v_{0, \mathrm{e}}}^{\infty} R_{\mathrm{e}}(v) f(v, T) \mathrm{d} v$

where $v_{0, \mathrm{e}}$ is the electron/ion velocity corresponding to the electronic dissociation energy $E_{0}$. The temperature $T$ is the same for both ions and electrons, but these latter will reach much larger velocities. From Eq. (21) we then expect to find a significantly higher collision rate with respect to the ion case.

\section{Results}

\subsection{PAH destruction in a hot gas}

To describe the destructive effects on PAHs of collisions with ions $i(i=\mathrm{H}, \mathrm{He}, \mathrm{C})$ and electrons in a thermal gas we have to evaluate the rate constant for carbon atom loss.

For all processes the rate constant $J$ is defined by the ratio $J=R_{\mathrm{T}} / n_{\mathrm{H}}$, where $R_{\mathrm{T}}$ is the thermal collision rate appropriate for nuclear and electronic excitation and electron collisions (cf. Eqs. (8) and (23)), and $n_{\mathrm{H}}$ is the density of hydrogen nuclei. For electronic and electron interactions the rate must be multiplyied by a factor of 2 , to take into account the fact that each collision leads to the loss of two carbon atoms.

The electron, nuclear and electronic rate constants for three PAH sizes $(\mathrm{Nc}=50,100$ and $200 \mathrm{C}$-atom) are shown in Fig. 5 as a function of the gas temperature. We assume for the nuclear threshold energy $T_{0}$ the value of $7.5 \mathrm{eV}(\mathrm{MJT})$, and for the electronic dissociation energy the value $4.58 \mathrm{eV}$ (Sect. 4.1).

For the nuclear interaction, the threshold energy $T_{0}$ is independent from the PAH size, so the three curves start at the same temperature (not shown in the plot). The small separation between the curves is due to the fact that the PAH "surface area" and hence the rate constant - scales linearly with $N_{\mathrm{C}}$, therefore $J$ is higher for bigger PAHs.

For nuclear (and electronic) interactions, the rate constants decrease from hydrogen to carbon because of the lower abundance of the heavier projectiles with respect to hydrogen $\left(\mathrm{H}: \mathrm{He}: \mathrm{C}=1: 0.1: 10^{-4}\right)$. On the other hand, the nuclear curves shift toward lower temperatures from lighter to heavier projectiles. This is the reflection of the fact that the critical energy of the particle, required to transfer the threshold energy $T_{0}$, decreases with increasing mass of the projectile itself. Then, a carbon atom with a temperature of $\sim 4 \times 10^{3} \mathrm{~K}$ is hot enough to transfer the energy required for atom removal via nuclear interaction, whereas for hydrogen a temperature of at least $10^{4} \mathrm{~K}$ is necessary. The almost-constant behaviour after the initial rise reflects the large maximum observed in the nuclear cross section (cf. Fig. 2 in MJT).

The dissociation probability $P\left(n_{\max }\right)$ (Eq. (17)) depends on the binding energy of the fragment $E_{0}$, on the PAH size $N_{\mathrm{C}}$ and on the energy transferred (through $T_{\mathrm{av}}$ ), which in turns depends on the initial energy (velocity) of the projectile. For a thermal distribution, this latter will be determined by the gas temperature $T$.

The electronic rate constant curves for the different PAH sizes are well separated at the lowest temperatures. This reflects the fact that, for a fixed value of the transferred energy and of the electronic dissociation energy $E_{0}$, the dissociation probability decreases for increasing $N_{\mathrm{C}}$ because either more energy is 
E. R. Micelotta et al.: PAH processing in a hot gas
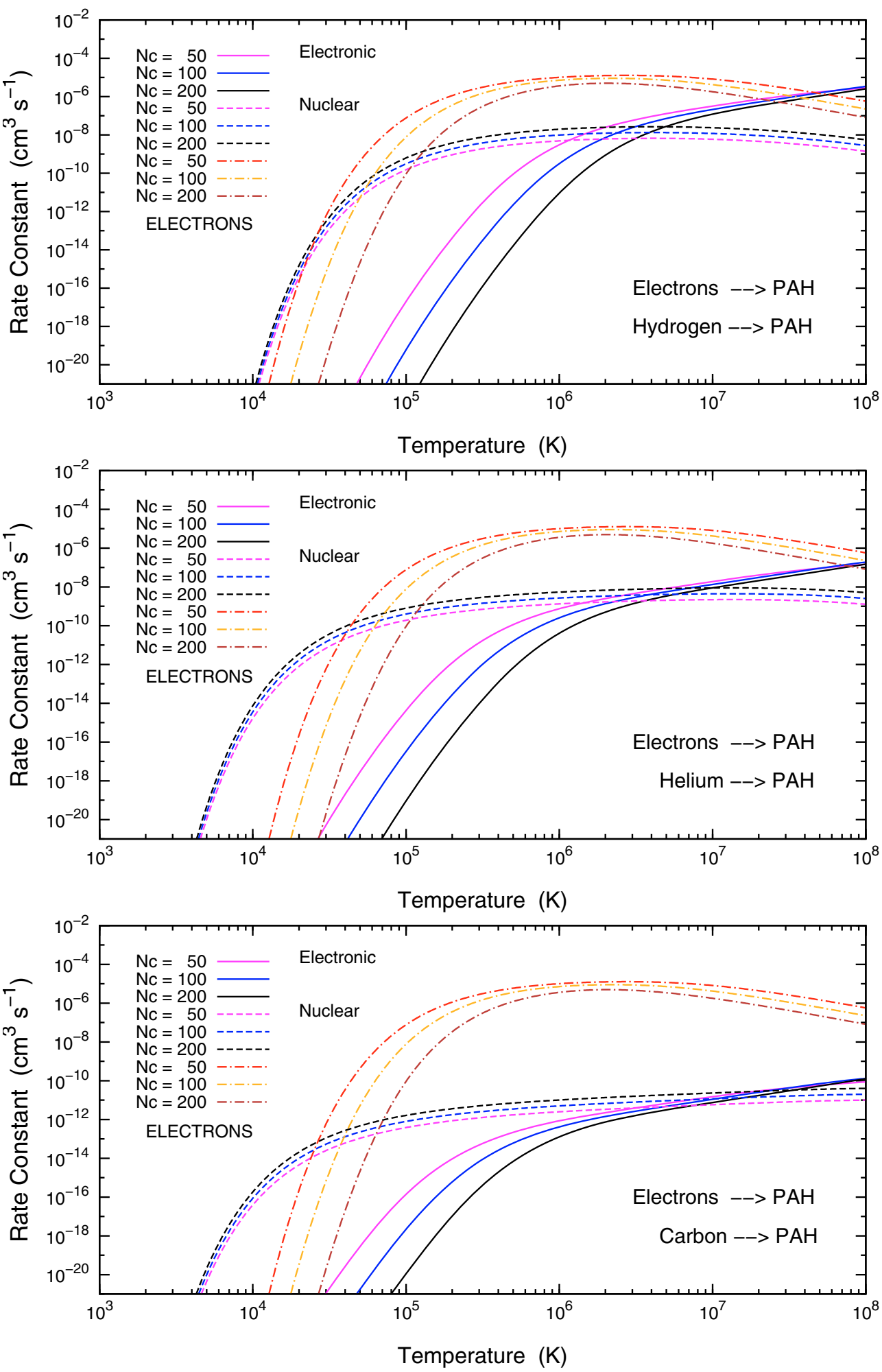

Fig. 5. Nuclear (dashed lines), electronic (solid lines) and electron (dashed-dotted lines) rate constant for PAH carbon atom loss due to collisions with $\mathrm{H}, \mathrm{He}, \mathrm{C}$ and electrons in a thermal gas. The rate constants are calculated as a function of the gas temperature for three PAH sizes $N_{\mathrm{C}}=50$, $100,200 \mathrm{C}$-atoms, assuming the nuclear threshold energy $T_{0}=7.5 \mathrm{eV}$ and the electronic dissociation energy $E_{0}=4.58 \mathrm{eV}$.

required in the bond that has to be broken or because the energy is spread over more vibrational modes and hence the internal excitation temperature is lower. On the other hand, the more energy that is deposited into the PAH, the higher is the dissociation probability. The energy transferred via electronic excitation (and then $T_{\mathrm{av}}$ ) increases with the energy of the projectile up to a maximum value, corresponding to an incident energy of $100 \mathrm{keV}$ for $\mathrm{H}$ (and higher for more massive particles), and decreases beyond that for higher energies. The energy content of a thermal gas at $T=10^{8} \mathrm{~K}$ is $\sim 9 \mathrm{keV}$, thus, in the temperature range considered in this study, the energy transferred increases with temperature (energy) and hence the dissociation 
probability increases as well. This is the basis for the monotonic rise of the electronic rate constant. After the initial separation, the three curves converge, because the rise in the transferred energy compensates the effect of increasing $N_{\mathrm{C}}$.

As discussed in Sect. 3, the energy transferred by impacting electrons rises sharply for energies in excess of $10 \mathrm{eV}$, peaks at $\sim 100 \mathrm{eV}$ and decreases more slowly down to $10 \mathrm{keV}$. This results in a dissociation probability shaped as a step function: for $10 \mathrm{eV} \lesssim v \lesssim 4 \mathrm{keV}, P\left(n_{\max }\right)$ jumps from values close to zero up to 1 . These limiting energies apply to a $50 \mathrm{C}$-atom $\mathrm{PAH}$; for $N_{\mathrm{C}}=200$ the width of the step is smaller $(100 \mathrm{eV} \lesssim v \lesssim 1 \mathrm{keV})$, due to the fact that for a bigger PAH more energy has to be transferred for dissociation. This behaviour is reflected in the shape of the electron rate constant, where a steep rise is followed by a maximum, which is emphasised by the logarithmic scale used for the plot. As expected, the electron rate constant overcomes the electronic one, except for the highest gas temperatures. This results from the fact that, for a given temperature, electrons can reach higher velocities with respect to the ions (cf. Eqs. (21) and (23)).

To summarize, from Fig. 5 we can infer that, according to our model, the destruction process is dominated by nuclear interaction with helium at low temperatures (below $\sim 3 \times 10^{4} \mathrm{~K}$ ), and by electron collisions above this value. Small PAHs are easier destroyed than big ones for temperatures below $\sim 10^{6} \mathrm{~K}$, while the difference in the destruction level reduces significantly for hotter gas.

The calculated rate constants, shown in Fig. 5, are well fitted by the function $g(T)=10^{f(\log (T))}$, where $f(T)$ is a polynomial of order 5

$$
f(T)=a+b T+c T^{2}+d T^{3}+e T^{4}+f T^{5} .
$$

For each PAH size we provide the fit to the electron rate constant; for the ions, the fit is over the sum of the nuclear and electronic rate constants, in order to provide an estimate of the global contribution from ionic collisions. The fits are shown in Fig. 6, and the fitting parameters are reported in Table 2. To provide an example of the accuracy of our fitting procedure, Fig. 7 shows the comparison between the calculated rate constant and the corresponding analytical fit, for electrons and helium impacting on a $50 \mathrm{C}$-atom PAH. The He fit is over the sum of the nuclear and electronic rate constants. The average fitting discrepancy is $\sim 15 \%$ and the fits are therefore well within any uncertainties in the method.

Figure 8 shows the comparison between the carbon loss rate constants for a very big PAH, $N_{\mathrm{C}}=1000$, and a 50 C-atom molecule. We assume $T_{0}=7.5 \mathrm{eV}$ and $E_{0}=4.6 \mathrm{eV}$. Because of the decrease of the dissociation probability when the PAH size increases, as expected, the electron and electronic rate constants are strongly suppressed, and both curves shift toward higher temperatures. Indeed, for such a big $\mathrm{PAH}$, a much higher internal energy is required to reach the internal temperature where dissociation sets in. On the other hand, the nuclear rate constant increases linearly with the PAH size. As a result, for a 1000 carbon atoms $\mathrm{PAH}$, the nuclear interaction is the dominant (and efficient) destruction mechanism up to $T \sim 2 \times 10^{7} \mathrm{~K}$. In conclusion, electrons are responsible for the destruction of small/medium size $\mathrm{PAH}$, while for big molecules this role is taken by ions.

As mentioned at the end of Sect. 4.1, we examined the effects of adopting different values for the Arrhenius energy, $E_{0}=3.65$ and $5.6 \mathrm{eV}$, lower and higher, respectively, than our canonical value $4.6 \mathrm{eV}$. The results are shown in Fig. 9. The dissociation probability decreases for increasing $E_{0}$, because more energy is required in the bond that has to be broken. Hence, as expected, both the electron and electronic rate constants decrease in absolute value and shift toward highest temperatures. In particular the electronic thermal shift is very pronounced, indicating how sensitive this process is with respect to the assumed $E_{0}$. A variation in the adopted electronic excitation energy translates into a significant variation of the rate constant, reemphasizing the importance of experimental studies of this critical energy.

\subsection{PAH lifetime}

Under the effect of electron and ion collisions in a hot gas, the number of carbon atoms in a PAH molecule varies with time. After a time $t$, this number is

$N_{\mathrm{C}}(t)=N_{\mathrm{C}}(0) \exp \left[-t / \tau_{0}\right]$

and the number of carbon atoms ejected from this PAH is

$F_{\mathrm{L}}(t)=\left(1-\exp \left[-t / \tau_{0}\right]\right)$.

The quantity $\tau_{0}$ is the time constant appropriate for electron, nuclear and electronic interaction, given by

$\tau_{0}=\frac{N_{\mathrm{C}}}{(2) R_{\mathrm{T}}}=\frac{N_{\mathrm{C}}}{J n_{\mathrm{H} / \mathrm{e}}}$

where $R_{\mathrm{T}}$ and $J$ are the thermal collision rate and the rate constant for electrons, nuclear and electronic interactions respectively, and $n_{\mathrm{H} / \mathrm{e}}$ is the hydrogen/electron density. For electrons and electronic excitation, the rate must be multipliyed by a factor of 2, because each interaction leads to the removal of two carbon atoms from the PAH. The nuclear rate scales linearly with $N_{\mathrm{C}}$, hence the corresponding fractional carbon atom loss $F_{\mathrm{L}}$ is independent of PAH size.

For any given incoming ion and fixed PAH size $N_{\mathrm{C}}, F_{\mathrm{L}}$ is univocally determined by the hydrogen/electron density $n_{\mathrm{H} / \mathrm{e}}$ and the gas temperature $T$. We assume that a PAH is destroyed after the ejection of $2 / 3$ of the carbon atoms initially present in the molecule. This occurs after the time $\tau_{0}$ (Eq. (27)) which we adopt as the PAH lifetime against electron and ion bombardment in a gas with given density and temperature.

Table 3 summarizes relevant data and PAH lifetime, $\tau_{0}$, for four objects characterized by warm-to-hot gas, X-ray emission, and (bright) IR emission features. The lifetime has been calculated for PAHs with $N_{\mathrm{C}}=200 . \tau_{0}$ (ref) has been evaluated adopting our reference values for the interaction parameters, $E_{0}=4.6 \mathrm{eV}$ and $T_{0}=7.5 \mathrm{eV}, \tau_{0}(\min )$ corresponds to the minimum values $E_{0}=3.65 \mathrm{eV}$ and $T_{0}=4.6 \mathrm{eV}$, and $\tau_{0}(\max )$ to the maximum values $E_{0}=5.6 \mathrm{eV}$ and $T_{0}=15 \mathrm{eV}$. $\tau_{\text {object }}$ is the lifetime of the object.

Clearly, PAHs or related larger species can survive in these environments. Figure 10 shows the fractional $\mathrm{C}$-atom loss, due to electron and ion $(\mathrm{H}+\mathrm{He}+\mathrm{C})$ collisions, for two widely different objects: the Orion Nebula (M42) and the M 82 galaxy (cf. Table 3). The famous Orion Nebula is an $\mathrm{H}$ II region with high density $\left(n_{\mathrm{H}}=10^{4} \mathrm{~cm}^{-3}\right)$ and low temperature $(T=7000 \mathrm{~K})$ gas, while M 82 is a starburst galaxy, which shows outside the galactic plane, a spectacular bipolar outflow of hot and tenuous gas $\left(n_{\mathrm{H}}=0.013 \mathrm{~cm}^{-3}, T=5.8 \times 10^{6} \mathrm{~K}\right)$.

In M 82, PAHs are completely destroyed by electrons, even for the larger PAH, before electronic and nuclear contributions start to be relevant. The electron and electronic fractional losses decrease with $\mathrm{PAH}$ size, thus bigger molecules can survive longer, while the nuclear loss is independent of $N_{\mathrm{C}}$. The destruction timescale is very short: after one thousand years the PAHs should have completely disappeared. 
E. R. Micelotta et al.: PAH processing in a hot gas
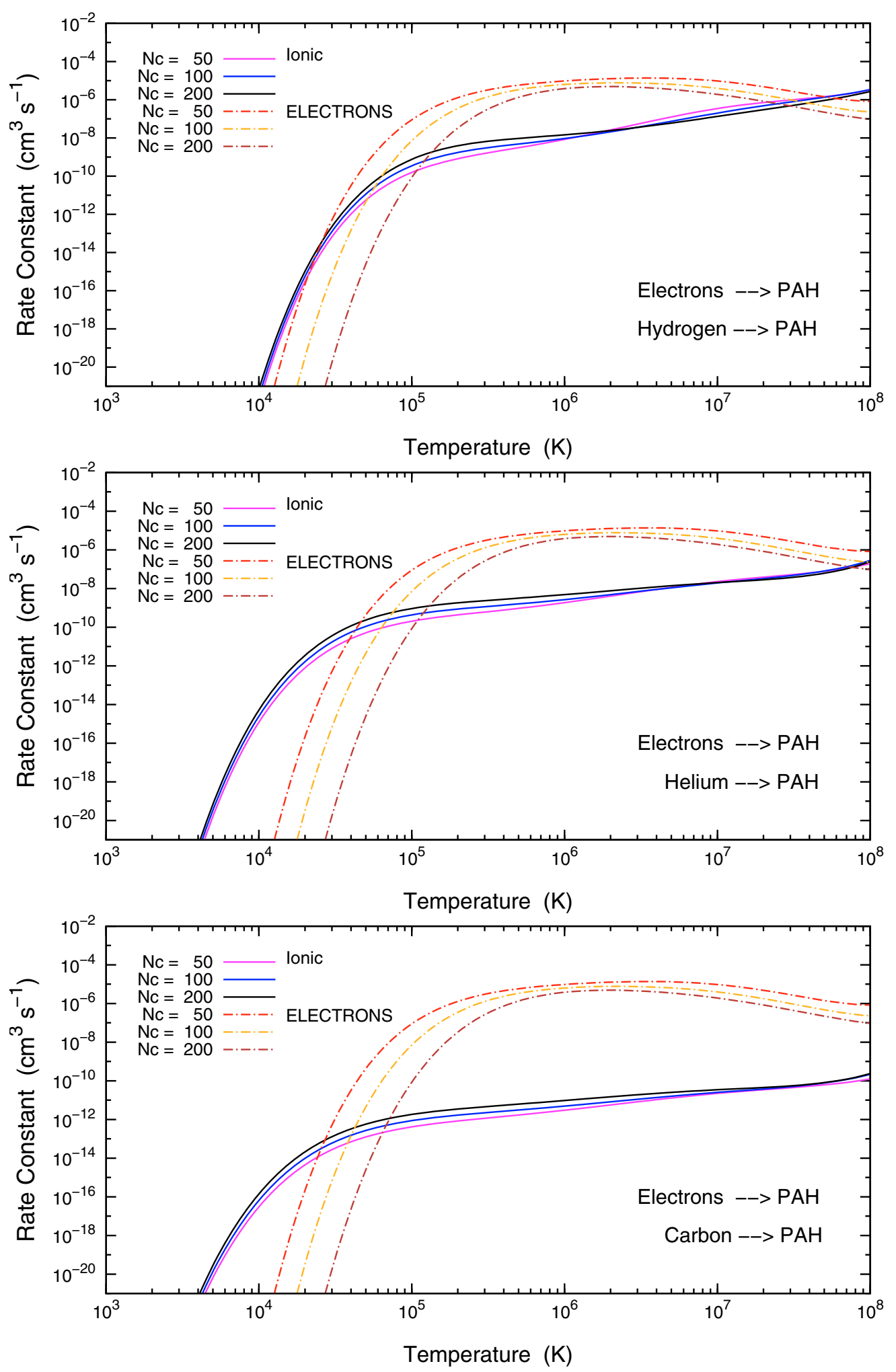

Fig. 6. Analytical fits to the calculated rate constants shown in Fig. 5. The "Ionic" curve is the fit to the sum of the nuclear and electronic rate constants, thus represents the total contribution from ion collisions to PAH destruction.

In Orion the situation is reversed. At the temperature considered for this object, electrons and electronic excitation do not contribute to PAH erosion (cf. Fig. 5). The damage is caused by nuclear interaction due to He collisions, with a marginal contribution from carbon because of low abundance, and the timescale is much larger: only after 10 million years the PAH destruction becomes relevant. Of course, we have not evaluated the destruction of PAHs by H-ionizing photons in the Orion H II region, which is expected to be very important.

The young ( $\simeq 2500 \mathrm{yr})$ supernova remnant, N132D, in the Large Magellanic Clouds has been studied in detail at IR, optical, UV, and X-ray wavelengths (Morse et al. 1995; Tappe et al. 2006). A Spitzer/IRS spectrum of the Southern rim shows evidence for the 15-20 $\mu$ m plateau - often attributed to large PAHs 


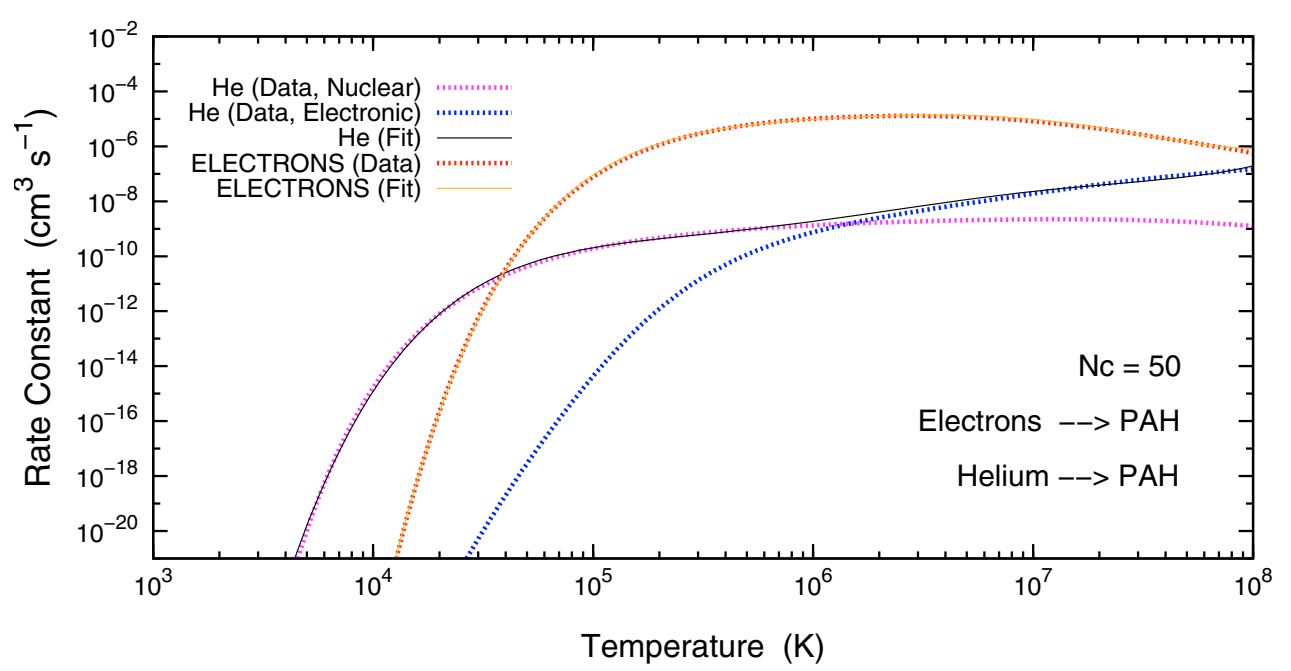

Fig. 7. Calculated rate constants for electrons and helium impacting on a $50 \mathrm{C}$-atom PAH, overlaid are the corresponding analytical fits. The He fit is for the sum of the nuclear and electronic rate constants. The average discrepancy is $\sim 15 \%$.

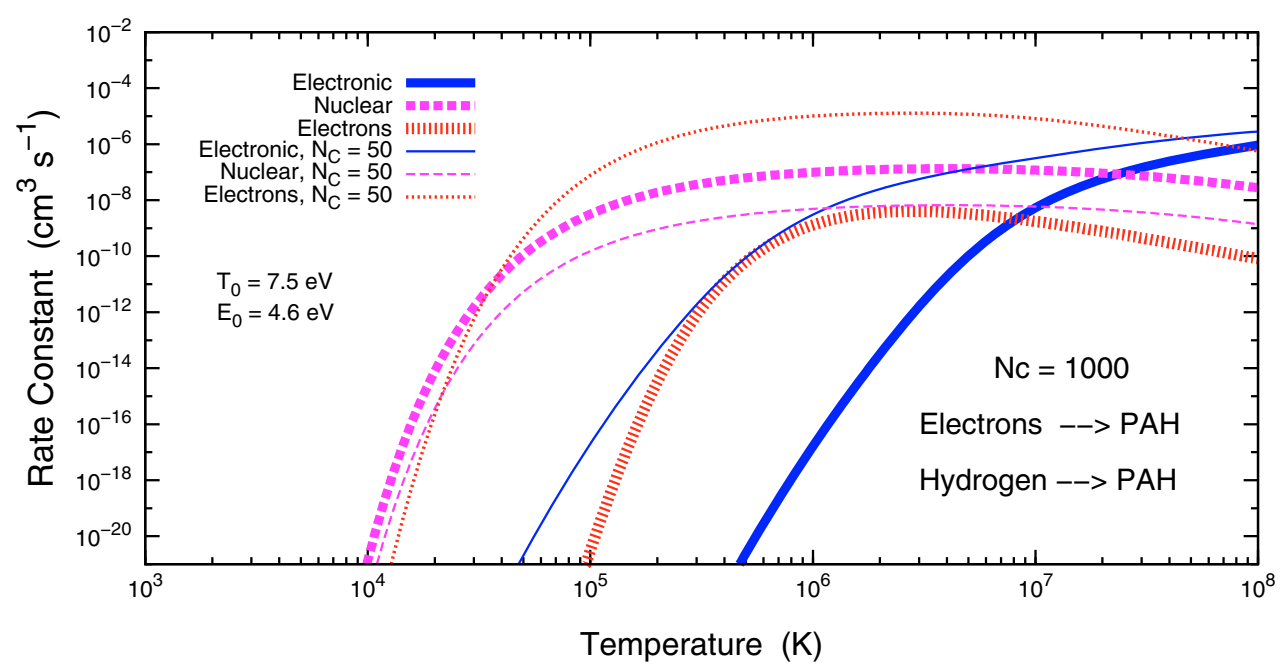

Fig. 8. Carbon atom loss rate constant for electrons and hydrogen impacting against a $1000 \mathrm{C}$-atom PAH. The rate constants for $N_{\mathrm{C}}=50$ are shown for comparison.

Table 2. Analytical fit parameters for the PAH carbon atom loss rate constant, calculated for electron and ion collisions.

\begin{tabular}{cccccccc}
\hline \hline \multicolumn{6}{c}{ Fitting function } & \multicolumn{6}{c}{$f(T)=a+b T+c T^{2}+d T^{3}+e T^{4}+f T^{5}$} \\
\hline & & $a$ & $b$ & $c$ & $d$ & $e$ & $f$ \\
\hline \multirow{5}{*}{$N_{\mathrm{C}}=50$} & Electrons & 2136.83 & 1632.17 & -499.822 & 76.4347 & -5.82964 & 0.177174 \\
& $\mathrm{H}$ & -1896.69 & 1480.8 & -462.733 & 71.8957 & -5.54719 & 0.169996 \\
& $\mathrm{He}$ & -971.448 & 770.259 & -245.561 & 38.8995 & -3.05787 & 0.0954303 \\
& $\mathrm{C}$ & -704.392 & 551.313 & -175.063 & 27.6506 & -2.16871 & 0.0675643 \\
\hline \multirow{5}{*}{$N_{\mathrm{C}}=100$} & Electrons & -2255.38 & 1681.45 & -503.451 & 75.4339 & -5.64956 & 0.168959 \\
& $\mathrm{H}$ & -1645.64 & 1257.06 & -384.309 & 58.4103 & -4.41007 & 0.132356 \\
& $\mathrm{He}$ & -945.901 & 747.921 & -237.984 & 37.6808 & -2.96613 & 0.0928852 \\
& $\mathrm{C}$ & -711.244 & 558.48 & -177.983 & 28.2547 & -2.23145 & 0.0701374 \\
\hline \multirow{5}{*}{$N_{\mathrm{C}}=200$} & Electrons & -2234.37 & 1597.71 & -459.647 & 66.332 & -4.79841 & 0.139019 \\
& $\mathrm{H}$ & -1473.64 & 1109.01 & -334.292 & 50.1417 & -3.74133 & 0.111164 \\
& $\mathrm{He}$ & -963.188 & 765.639 & -245.054 & 39.0745 & -3.10123 & 0.0980047 \\
& $\mathrm{C}$ & -738.791 & 584.928 & -187.884 & 30.0819 & -2.39748 & 0.0760639 \\
\hline
\end{tabular}

or PAH clusters (Van Kerckhoven et al. 2000; Peeters et al. 2004) - and, tentatively, weak PAH emission features near 6.2, 7.7, and $11.2 \mu \mathrm{m}$ (Tappe et al. 2006). Tappe et al. (2006) attribute these features to emission from large ( $4000 \mathrm{C}$-atom) PAHs either just swept up by the blast wave and not yet completely destroyed by the shock or in the radiative precursor of the shock. We calculate a lifetime of small (50-200 C-atom) PAHs in the relatively dense, hot gas of this young supernova remnant of $\sim 4$ months (Table 3). In contrast, we estimate a lifetime of $150 \mathrm{yr}$ for $4000 \mathrm{C}$-atom species (cf. Eq. (27) and Fig. 8). It is clear that 


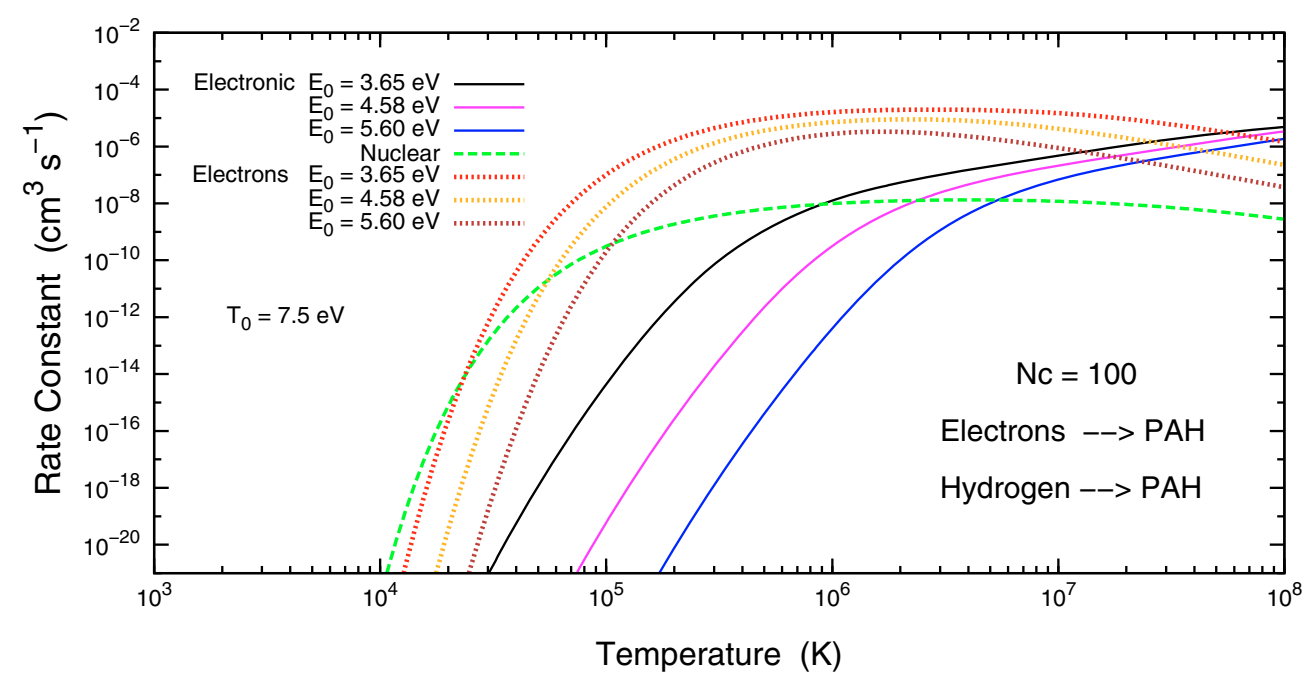

Fig. 9. Comparison between carbon atom loss rate constants calculated assuming three values for the electronic dissociation energy, $E_{0}: 3.65,4.58$ and $5.6 \mathrm{eV}$. The curves refer to electrons and hydrogen impacting against a $100 \mathrm{C}$-atom PAH. The nuclear rate constant is calculated assuming $T_{0}=7.5 \mathrm{eV}$.

Table 3. Physical properties and PAH lifetime, $\tau_{0}$, in objects showing PAH emission characteristics.

\begin{tabular}{ccccccccc}
\hline \hline Object & $\begin{array}{c}n_{\mathrm{H}} \\
\left(\mathrm{cm}^{-3}\right)\end{array}$ & $\begin{array}{c}T \\
\mathrm{~K}\end{array}$ & Reference & $\begin{array}{c}\tau_{0}(\mathrm{ref}) \\
(\mathrm{yr})\end{array}$ & $\begin{array}{c}\tau_{0}(\min ) \\
(\mathrm{yr})\end{array}$ & $\begin{array}{c}\tau_{0}(\max ) \\
(\mathrm{yr})\end{array}$ & $\begin{array}{c}\tau_{\text {object }} \\
(\mathrm{yr})\end{array}$ & $\begin{array}{c}\text { Destruction } \\
\text { agent }\end{array}$ \\
\hline M 82 & 0.013 & $5.8 \times 10^{6}$ & $(\mathrm{a})$ & 162 & 43 & 1302 & $\sim 2 \times 10^{7}$ & electrons \\
M 17 & 0.3 & $7 \times 10^{6}$ & (b) & 8.2 & 2 & 71 & $\sim 1 \times 10^{6}$ & electrons \\
N132D & 10 & $8 \times 10^{6}$ & (c) & 0.3 & 0.07 & 2.5 & $\sim 3 \times 10^{3}$ & electrons \\
Orion & $10^{4}$ & $7 \times 10^{3}$ & (d) & $1.3 \times 10^{7}$ & $1.4 \times 10^{5}$ & $7.5 \times 10^{11}$ & $\sim 1 \times 10^{6}$ & ions \\
\hline
\end{tabular}

References. (a) Ranalli et al. (2008); (b): Townsley et al. (2003); (c): Hwang et al. (1993); (d): Tielens (2005).

the PAH-grain size distribution will be strongly affected in this environment. Given advection of fresh material into the shocked hot gas, the observations are in reasonable agreement with our model expectations. We note that the observed shift to larger PAH sizes - so evident in the observations - implies that the emitting species are not associated with the precursor but are actualy tracing the postshock gas and are therefore likely heated through collisions with the hot electrons.

This discussion clearly shows that in a low density - high temperature gas, small PAHs are rapidly and completely destroyed. Survival of small PAHs in such environnement (and so the possibility to be detected) requires a protective environment and/or an efficient reformation mechanism.

\section{Discussion}

\subsection{X-ray absorption}

As our calculations show, PAHs are rapidly destroyed in the hot gas associated with stellar winds and supernova explosions. Any PAHs observed near such regions have to be isolated from this hot gas and are presumably in cold gas entrained in these stellar and galactic winds. However, such PAHs would still be exposed to energetic X-ray photons and these can be very destructive as well (Voit 1992; Boechat-Roberty et al. 2008).

The photon absorption cross section of PAHs shows strong peaks at about 6 and $17.5 \mathrm{eV}$ associated with transitions involving the $\pi$ and $\sigma$ electronic manifolds and then another broad peak around $286 \mathrm{eV}$ due to carbon K-shell transitions (Keller et al. 1992; de Souza et al. 2002; Regier et al. 2007). Each of these peaks can show various subpeaks due to electronic and vibrational structure. Here, we focus on the high energy peak. Because we are only concerned with the Planck-averaged cross section, all the fine detail will be washed out and we have elected to evaluate the X-ray absorption rate adopting the measured absolute cross section of graphitic carbon taken from the NIST data base (Chantler 1995, 2000). Figure 11 shows the (photon) averaged cross sections as a function of the black body temperature. As this figure illustrates, even the strong absorption edge due to the carbon $\mathrm{K}$-shell is washed out by this averaging process, justifying our neglect of the fine detail in the absorption cross sections of individual PAHs. Our averaged cross section is also in good agreement with the recent study of Boechat-Roberty et al. (2008) for benzene.

The photon absorption rate, $R_{\mathrm{X}}$, of a $\mathrm{PAH}$ exposed to an $\mathrm{X}$-ray photon flux, $N_{\mathrm{X}}$ is then given by,

$R_{\mathrm{X}}=\sigma_{\mathrm{X}}\left(T_{\mathrm{X}}\right) N_{\mathrm{X}}$

with $\sigma_{\mathrm{X}}\left(T_{\mathrm{X}}\right)$ the Planck average absorption cross section at temperature, $T_{\mathrm{X}}$. Consider now PAHs embedded in cold gas entrained in the stellar wind of the ionizing stars of the $\mathrm{H}$ II region M 17. The observed X-ray luminosity is $4 \times 10^{33} \mathrm{erg} \mathrm{s}^{-1}$ and the X-ray temperature is $7 \times 10^{6} \mathrm{~K}$ (Townsley et al. 2003). With a projected emitting area of $42 \mathrm{pc}^{2}$ this then implies an average X-ray photon intensity of $\simeq 2.6 \times 10^{3}$ photons $\mathrm{cm}^{-2} \mathrm{~s}^{-1}$. With an average cross section of $\sigma_{\mathrm{X}} \simeq 9 \times 10^{-20} \mathrm{~cm}^{2} / \mathrm{C}$-atom, we calculate a photon absorption rate of $7.2 \times 10^{-3}$ photons/Catom/Myr. After X-ray photon absorption, a PAH will typically lose a few $\mathrm{C}$-atoms and, hence, the estimated lifetime of PAHs entrained in the stellar winds from M 17 is $~ 50$ Myr. This is in excess of the stellar lifetime and the overall life time of the 

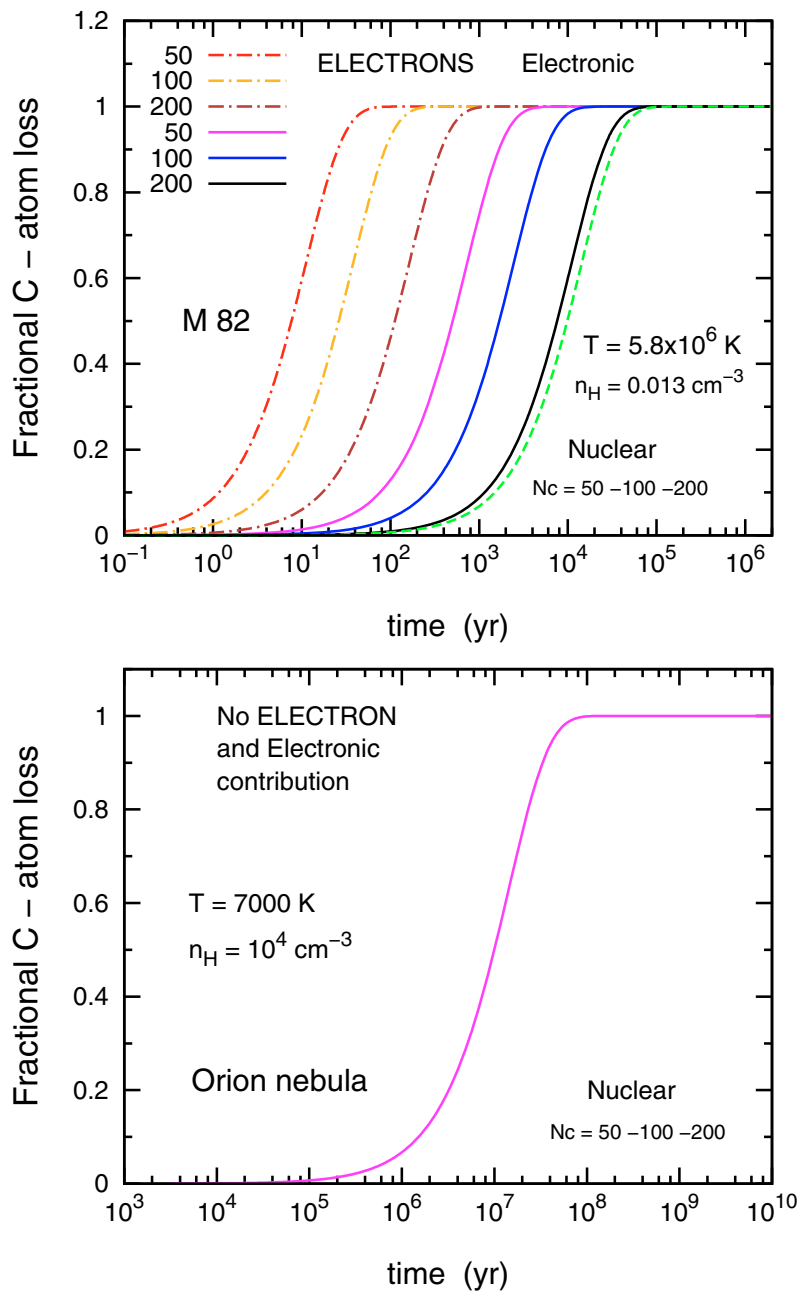

Fig. 10. Nuclear and electronic fractional C-atom loss as a function of time in the M 82 galaxy (top) and in the Orion Nebula (bottom), calculated for three PAH sizes $N_{\mathrm{C}}=50,100,200 \mathrm{C}$-atoms. The fractional carbon loss is defined as the number of carbon atoms ejected from the PAH after a given time divided by $N_{\mathrm{C}}$, and represents a direct indicator of the level of destruction of the PAH. Each curve shows the total contribution from collisions with $\mathrm{H}, \mathrm{He}$ and $\mathrm{C}$. In the nuclear case the fractional loss is independent from $N_{\mathrm{C}}$, so the three curves coincide, whereas for electrons and electronic interaction the fractional loss varies with the PAH size. In M 82 the PAH destruction is dominated by electron collisions and occurs on a very short timescale ( $\sim$ thousand years). In Orion instead, because of the low gas temperature no electron and electronic contribution are possible (the rate constant is close to zero, see Fig. 5), and hence only nuclear interaction is responsible for the PAH erosion, which however takes place on a long timescale (some $10^{7}$ years).

region. PAHs embedded in cold gas entrained in the galactic superwind of M 82 are exposed to somewhat harsher conditions. For M 82, at a distance of $3.6 \mathrm{Mpc}$, the observed soft $(0.5-2.0 \mathrm{keV}) \mathrm{X}$-ray flux is $10^{-11} \mathrm{erg} \mathrm{cm}^{-2} \mathrm{~s}^{-1}$ and the temperature is $7 \times 10^{6} \mathrm{~K}$. Evaluating the X-ray flux at a distance of $3 \mathrm{kpc}$ from the nuclear starburst region, we calculate a photon absorption rate of $1.8 \times 10^{-2}$ photons/C-atom/Myr. Hence, in this environment, PAHs would be destroyed on a timescale of 20 Myr, comparable to or larger than the starburst lifetime. It is clear that PAHs in the hot gas are predominantly destroyed by collisions with the hot gas and that X-ray photon absorption plays little role.

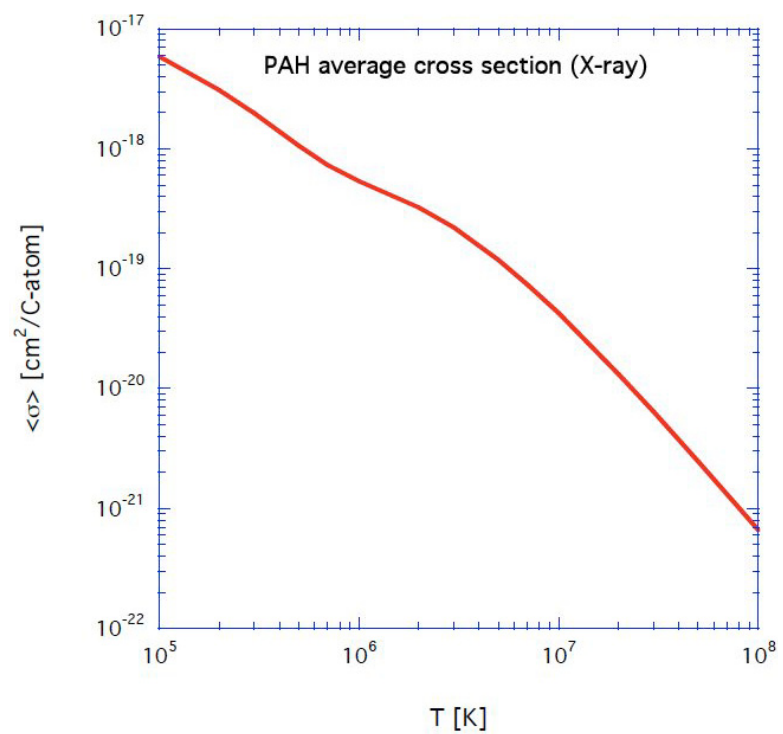

Fig. 11. Planck averaged cross section of PAHs as a function of temperature. The cross section is on a per $\mathrm{C}$-atom basis. The strong carbon $\mathrm{K}$ shell band edge at $\simeq 286 \mathrm{eV}\left(2 \times 10^{6} \mathrm{~K}\right)$ has been largely washed out by the averaging process.

Finally, we note that here we have assumed that every X-ray photon absorption will lead to photodissociation. Actually, $\mathrm{H}$-shell electron ejection will be rapidly followed by the Auger effect filling the K-shell again and simultaneously ejecting a second electron. This process may leave the PAH internally excited with typically $15-35 \mathrm{eV}^{2}$. This energy is well above the measured appearance energy of PAH fragmentation for small PAHs under radiative cooling conditions (cf., Sect. 4.1) but larger PAHs may survive. Indeed, for an Arrhenius energy of $4.6 \mathrm{eV}$, a $50 \mathrm{C}$-atom PAH requires about $24 \mathrm{eV}$ of internal energy to dissociate with a probability of $1 / 2$ (cf., Sect. 4.1). Hence, not every X-ray photon absorption will lead to fragmentation, particularly for large PAHs. Further experiments on larger PAHs are required to settle this issue.

\subsection{PAHs as tracers of cold entrained gas}

The results in the previous subsection illustrate that PAHs can survive in the surface regions of cold gas clouds embedded in a hot gas for tens to hundreds of million years. The expansion of a stellar or galactic wind in an inhomogeneous environment will in a natural way lead to the entrainment of cold gas clumps (Smith et al. 1984; Chevalier \& Clegg 1985; Hartquist \& Dyson 1993; Suchkov et al. 1994; Strickland \& Stevens 2000; Marcolini et al. 2005). The material in these clumps will only be mildly shocked - and PAH destruction by shocks is not expected as long as the shock velocity is less than about $100 \mathrm{~km} \mathrm{~s}^{-1}$ (MJT) - and can be transported with the wind while losing its identity only slowly, mainly through evaporation into the hot gas driven by thermal conduction. Multiwavelength observations of such winds support this global view and much of the soft X-ray and $\mathrm{O}$ VI absorbing and emitting gas is now thought to originate from the interaction interfaces between the tenuous hot gas and the cold dense clumps (Chevalier \& Clegg 1985; Heckman et al. 2002).

\footnotetext{
2 The resulting PAH dication will quickly recombine with electrons and Coulomb explosion is of no consequence for such large PAHs.
} 
A typical $0.5 \mathrm{keV} \mathrm{X}$-ray photon will penetrate a column of about $10^{22} \mathrm{H}$-atoms $/ \mathrm{cm}^{2}$ and hence the "PAH-bright" surfaces of such PDRs - which are typically only a column of $5 \times 10^{21} \mathrm{H}$-atoms $/ \mathrm{cm}^{2}$ thick - will be slowly depleted of PAHs on the timescale calculated in Sect. 6.1. However, at the same time, entrained clumps will slowly evaporate due to thermal conduction. Considering classical thermal conduction, the mass loss of a cloud due to evaporation into the hot gas is given by

$\dot{M} \simeq 1.3 \times 10^{-3}\left(\frac{R}{10 \mathrm{pc}}\right)\left(\frac{T}{6 \times 10^{6} \mathrm{~K}}\right)^{5 / 2} \quad M_{\odot} \mathrm{yr}^{-1}$

with $T$ the temperature of the hot gas and $R$ the radius of the clump (cf. Tielens 2005). Consider the galactic wind of M 82 and a typical structure with a size of $10 \mathrm{pc}$. Assuming thermal pressure equilibrium between the clump and the hot gas (e.g., ignoring ram pressure confinement which may actually be a factor of 10 larger), we estimate a density of $\simeq 800 \mathrm{~cm}^{-3}$ for a typical PDR temperature of $100 \mathrm{~K}$ for the galactic wind region of M 82 . The lifetime of such a clump is then,

$\tau_{\text {clump }} \simeq 40\left(\frac{n_{\mathrm{PDR}}}{10^{3} \mathrm{~cm}^{-3}}\right)\left(\frac{R}{10 \mathrm{pc}}\right)^{2}\left(\frac{6 \times 10^{6} \mathrm{~K}}{T}\right)^{5 / 2} \mathrm{Myr}$.

Hence, "fresh" material will continuously be advected to the clump surface where ambient FUV photons can excite it. Thus, we conclude that such clumps can survive for a long time and that, proviso illumination by FUV photons, PAHs will form an excellent "dye" for tracing the presence of cold entrained material; or more specifically, for the exposed surfaces of photodissociation regions.

\subsection{Comparison with previous studies}

Dwek et al. (1996) determines a sputtering rate for dust in a hot dust gas $\left(T \geq 10^{6} \mathrm{~K}\right)$ that can be used to derive the lifetime, $\tau$ of a grain of a given radius in a hot gas of a given density, i.e.

$\tau \approx \frac{10^{3}}{n_{\mathrm{H}}}\left(\frac{a}{1 \AA}\right) \mathrm{yr}$,

where $a$ is the grain size and $n_{\mathrm{H}}$ is the gas density. Clearly in this situation it is the smallest grain that suffer the fastest destruction. Hence, a treatment of the small grains, including PAHs, really needs to take into account the detailed physics of the interactions. For illustrative purposes, we can use Eq. (31) to show that grains with sizes typical of PAHs ( $a \approx 5 \AA$ ) should have lifetimes, in a tenuous hot gas $\left(n_{\mathrm{H}}=1 \mathrm{~cm}^{-3}\right)$, of the order of at least a few thousand years. We can see that this timescale is much longer than the typical PAH lifetimes that we derive here, i.e. few years for a 50 carbon atom PAH in a gas with $n_{\mathrm{H}}=1 \mathrm{~cm}^{-3}$. The timescale determination arrived at using Eq. (31) assumes that a PAH just behaves as a small grain. However, in the case of a grain, the sputtering yield is usually much less that unity because atoms displaced by knock-on collisions in the solid can remain in the solid. The very much shorter lifetimes for the PAHs that we find can be ascribed to the fact that the transferred energy in incident electron and ion interactions always leads to the loss of carbon atoms from the PAH when that energy exceeds the relevant binding energy. In fact the equivalent PAH "sputtering yield" can be greater than unity because multiple atom ejection is possible. Indeed we assume here that the ejected species in the case of electronic interactions are $\mathrm{C}_{2}$ units.

The evolution of the dust and PAH thermal emission arising from a hot gas is affected by the changing dust size distribution.
As pointed out by Dwek et al. (1996), it is the short wavelength emission, coming from the smallest grains, that is most affected by dust destruction. Our work now indicates that the destruction timescale is much shorter than that predicted by Dwek et al. because the erosion of the smallest grains and PAHs is enhanced by as much as three orders of magnitude compared to the earlier work. Thus, the gas cooling rates derived by Dwek (1987) will need to be reduced if a significant fraction of the dust mass is in the form of small grains and PAHs.

\section{4. $C_{2}$ groups loss}

The primary channel for PAH erosion in a hot gas is the progressive loss of $\mathrm{C}_{2} \mathrm{H}_{n}(n=0,1,2)$ units following incident electron and ion excitation of the molecule. We note that this can only really occur from the periphery of the PAH (see the lower part of Fig. 11 in MJT for an illustration of this type of erosion). In this case we can see that a coherent aromatic structure will tend to be preserved, which is probably not the case where the inertial sputtering of $\mathrm{C}$ atoms is dominant. Thus, we conclude that $\mathrm{PAH}$ erosion in a hot gas will tend to preserve the aromatic structure throughout the destruction process.

As emphasized in MJT, PAH destruction may start with complete H-loss followed by isomerization to much more stable pure carbon clusters such as fullerenes (cf. Tielens 2008). While we have not assessed this point, we expect that such species will be more stable than PAHs in a hot gas.

\section{Conclusions}

We have extensively studied the stability of PAHs against electron and ion collisions ( $\mathrm{H}, \mathrm{He}$ and $\mathrm{C}$ ) in a hot gas, such as the gas behind fast non-radiative shocks and in galactic outflows. Collisions can lead to carbon atom loss, with a consequent disruption and destruction of the molecule. We consider the case of a thermal gas with temperature $T$ in the range $10^{3}-10^{8} \mathrm{~K}$.

An ionic collision consists of two simultaneous processes which can be treated separately: a binary collision between the projectile ion and a single atom in the target (nuclear interaction) and energy loss to the electron cloud of the molecule (electronic interaction).

For the nuclear interaction, as described in MJT, we have modified the existing theory in order to treat collisions able to transfer energy above a specific threshold $T_{0}$. We adopt $T_{0}=$ $7.5 \mathrm{eV}$ as a reasonable value, but experimental determinations of this quantity are necessary.

The electronic energy transfer has been described in term of the stopping power of an electron gas with appropriate electron density (jellium approximation). For electron collisions, we derived an analytical expression for the measured electron stopping power in graphite and applied this to the case of PAHs.

The dissociation probability for a PAH excited by electronic interactions and electron collisions, has been derived using the theory of unimolecular reactions. The parameter $E_{0}$, which governs the dissociation probability, is not well constrained. We adopt a value of $4.6 \mathrm{eV}$ consistent with extrapolations to interstellar conditions but better determinations, relevant to the astrophysical situation, are needed.

The PAH destruction process is dominated by electron collisions for gas temperatures above $\sim 3 \times 10^{4} \mathrm{~K}$, and by nuclear interaction with helium below this value. Small PAHs are more easily destroyed than larger ones below $\sim 10^{6} \mathrm{~K}$, while the difference reduces significantly for a hotter gas. For a $1000 \mathrm{C}$-atom $\mathrm{PAH}$, nuclear interactions are the dominant destruction process. 
In a hot and tenuous gas (e.g. M 82 galactic outflows), PAHs with sizes between 50 and $200 \mathrm{C}$-atom are destroyed by electron collisions in few thousand years. In denser and colder regions (e.g. Orion), PAHs can survive for some $10^{7} \mathrm{yr}$ before being destroyed by nuclear interaction processes.

$\mathrm{X}$-ray photon absorption plays little role in PAH destruction in the hot gas associated with stellar winds and supernova explosions, with respect to electron collisions. The PAH destruction process in a hot gas is therefore dominated by electron collisions. Any PAHs observed near such regions have to be isolated from this hot gas and are presumably in a cooler PDR-type gas entrained in the stellar and galactic winds. In this sense, PAHs represent a good tracer for the presence of entrained denser material.

Our calculated PAH lifetime in a hot tenuous gas $\left(T \sim 10^{6} \mathrm{~K}\right.$, $\left.n_{\mathrm{H}}=1 \mathrm{~cm}^{-3}\right)$, is much shorter than the lifetime of an equivalent dust grain of roughly the same size ( $a \approx 5 \AA$ ). Thus, might then imply that the destructive effects of ion and electron collisions with very small grains have previously been underestimated. The enhanced erosion of the smallest grain and PAHs implies that the gas cooling rates for a hot gas $\left(T>10^{6} \mathrm{~K}\right)-$ which depend on the grain/PAH size distribution - may need to be reduced if a significant fraction of the dust mass is locked in small grains and PAHs.

PAH erosion in a hot gas occurs mainly through the ejection of $\mathrm{C}_{2}$ groups following electron collisions and electronic excitation. The $\mathrm{C}_{2}$ loss occurs at the periphery of the molecule, thus the aromatic structure will tend to be preserved throughout the destruction process.

Acknowledgements. We are grateful to L. Allamandola and L. Verstraete for useful discussions, and we acknowledge our referee Tom Hartquist for careful reading and helpful comments. E.R.M. thanks G. Lavaux for support and technical assistance and acknowledges financial support by the EARA Training Network (EU grant MEST-CT-2004-504604).

\section{References}

Beirão, P., Brandl, B. R., Appleton, P. N., et al. 2008, ApJ, 676, 304

Bethe, H. 1930, Ann. Physik, 5, 325

Boechat-Roberty, H. M., Neves, R., Pilling, S., Lago, A. F., \& de Souza, G. G. B. 2008, ArXiv e-prints

Chantler, C. T. 1995, J. Phys. Chem. Ref. Data, 24, 71

Chantler, C. T. 2000, J. Phys. Chem. Ref. Data, 29(4), 597

Chevalier, R. A., \& Clegg, A. W. 1985, Nature, 317, 44

Czyzewski, Z., O’Neill MacCallum, D., Romig, A., \& Joy, D. C. 1990, J. Appl. Phys., 68, 3066

de Souza, G. G. B., Boechat-Roberty, H. M., Rocco, M. L. M., \& Lucas, C. A. 2002, J. Electron Spec. Rel. Phen., 123, 315

Désert, F.-X., Boulanger, F., \& Puget, J. L. 1990, A\&A, 237, 215

Draine, B. T., \& Li, A. 2001, ApJ, 551, 807

Draine, B. T., \& Salpeter, E. E. 1979, ApJ, 231, 438

Dwek, E. 1987, ApJ, 322, 812
Dwek, E., Foster, S. M., \& Vancura, O. 1996, ApJ, 457, 244

Engelbracht, C. W., Kundurthy, P., Gordon, K. D., et al. 2006, ApJ, 642, L127

Ferrell, T. 1979, Solid State Commun., 32, 419

Galliano, F., Madden, S. C., Tielens, A. G. G. M., Peeters, E., \& Jones, A. P. 2008, ApJ, 679, 310

Hadjar, O., Hoekstra, R., Morgenstern, R., \& Schlathölter, T. 2001, Phys. Rev. A, 63, 033201

Hartquist, T. W., \& Dyson, J. E. 1993, QJRAS, 34, 57

Heckman, T. M., Norman, C. A., Strickland, D. K., \& Sembach, K. R. 2002, ApJ, 577, 691

Hovington, P., Drouin, D., \& Gauvin, R. 1997, Scanning, 19, 1

Hwang, U., Hughes, J. P., Canizares, C. R., \& Markert, T. H. 1993, ApJ, 414, 219

Jochims, H. W., Ruhl, E., Baumgartel, H., Tobita, S., \& Leach, S. 1994, ApJ, 420, 307

Jones, A. P., Tielens, A. G. G. M., Hollenbach, D. J., \& McKee, C. F. 1994, ApJ, 433, 797

Jones, A. P., Tielens, A. G. G. M., \& Hollenbach, D. J. 1996, ApJ, 469, 740

Joy, D. C. 1995, Scanning, 17, 270

Joy, D. C., \& Luo, S. 1989, Scanning, 11, 176

Keller, J. W., Coplan, M. A., \& Goruganthu, R. 1992, ApJ, 391, 872

Le Page, V., Snow, T. P., \& Bierbaum, V. M. 2001, ApJS, 132, 233

Lindhard, J., Scharff, M., \& Schiott, H. E. 1963, Mat. Fys. Medd. Dan. Vid. Selsk., 33, No. 14

Ling, Y., \& Lifshitz, C. 1998, JPC A, 102, 708

Marcolini, A., Strickland, D. K., D’Ercole, A., Heckman, T. M., \& Hoopes, C. G. 2005, MNRAS, 362, 626

Micelotta, E. R., Jones, A. P., \& Tielens, A. G. G. M. 2010, A\&A, 510, A36 (MJT)

Morse, J. A., Winkler, P. F., \& Kirshner, R. P. 1995, AJ, 109, 2104

Mott, N. F., \& Massey, H. S. W. 1949, The theory of atomic collisions, ed. N. F. Mott, \& H. S. W. Massey (Oxford: Clarendon Press)

Omont, A. 1986, A\&A, 164, 159

Peeters, E., Mattioda, A. L., Hudgins, D. M., \& Allamandola, L. J. 2004, ApJ, 617, L65

Povich, M. S., Stone, J. M., Churchwell, E., et al. 2007, ApJ, 660, 346

Puska, M. J., \& Nieminen, R. M. 1983, Phys. Rev. B, 27, 6121

Puska, M. J., \& Nieminen, R. M. 1993, Phys. Rev. A, 47, 1181

Ranalli, P., Comastri, A., Origlia, L., \& Maiolino, R. 2008, MNRAS, 386, 1464

Reach, W. T., Rho, J., Tappe, A., et al. 2006, AJ, 131, 1479

Regier, T. Z., Shi, J., Tersingi, A., et al. 2007, Canadian Light Source, 51

Schlathölter, T., Hadjar, O., Hoekstra, R., \& Morgenstern, R. 1999, Phys. Rev. Lett., 82, 73

Sigmund, P. 1981, Sputtering by Particle Bombardment Vol. 1, ed. R. Behrisch, in Topics in Applied Physics (Springer-Verlag), 47

Smith, L. J., Pettini, M., Dyson, J. E., \& Hartquist, T. W. 1984, MNRAS, 211, 679

Strickland, D. K., \& Stevens, I. R. 2000, MNRAS, 314, 511

Suchkov, A. A., Balsara, D. S., Heckman, T. M., \& Leitherer, C. 1994, ApJ, 430, 511

Tappe, A., Rho, J., \& Reach, W. T. 2006, ApJ, 653, 267

Tielens, A. G. G. M. 2005, The Physics and Chemistry of the Interstellar Medium (University of Cambridge Press)

Tielens, A. G. G. M. 2008, ARA\&A, 46, 289

Tomita, S., Andersen, J. U., Gottrup, C., Hvelplund, P., \& Pedersen, U. V. 2001, Phys. Rev. Lett., 87, 073401

Townsley, L. K., Feigelson, E. D., Montmerle, T., et al. 2003, ApJ, 593, 874

Van Kerckhoven, C., Hony, S., Peeters, E., et al. 2000, A\&A, 357, 1013

Voit, G. M. 1992, MNRAS, 258, 841 